\title{
Developmental decrease of entorhinal gate disrupts prefrontal-hippocampal communication in immune- challenged DISC1 knockdown mice
}

\section{Xiaxia Xu}

University Medical Center Hamburg-Eppendorf

Lingzhen Song

University Medical Center Hamburg-Eppendorf

Rebecca Kringel

University Medical Center Hamburg-Eppendorf

lleana Hanganu-Opatz ( $\nabla$ hangop@zmnh.uni-hamburg.de )

University Medical Center Hamburg-Eppendorf

\section{Article}

Keywords: prefrontal-hippocampal dysfunction, prefrontal-hippocampal communication

Posted Date: March 10th, 2021

DOI: https://doi.org/10.21203/rs.3.rs-290304/v1

License: (c) This work is licensed under a Creative Commons Attribution 4.0 International License.

Read Full License

Version of Record: A version of this preprint was published at Nature Communications on November 23rd, 2021. See the published version at https://doi.org/10.1038/s41467-021-27114-w. 


\title{
Developmental decrease of entorhinal gate disrupts prefrontal-hippocampal communication in immune- challenged DISC1 knockdown mice
}

\author{
Xiaxia Xu*, Lingzhen Song, Rebecca Kringel, and Ileana L. Hanganu-Opatz* \\ Institute of Developmental Neurophysiology, Center for Molecular Neurobiology, University \\ Medical Center Hamburg-Eppendorf, 20251 Hamburg, Germany \\ Running title: Abnormal entorhinal gate of developing circuits \\ ${ }^{*}$ Corresponding authors: Ileana L. Hanganu-Opatz \\ hangop@zmnh.uni-hamburg.de \\ Xiaxia Xu \\ xiaxia.xu@zmnh.uni-hamburg.de
}

Figures: 7

Tables: 1

Number of pages: 40

Number of words in Abstract: 150

Number of words in Introduction: 789

Number of words in Discussion: 954 


\section{Abstract}

32 The prefrontal-hippocampal dysfunction that underlies cognitive deficits in mental disorders emerges during early development. The contribution of the lateral entorhinal cortex (LEC), a gatekeeper of prefrontal cortex (PFC) and hippocampus (HP), to the early dysfunction is fully unknown. Here we show that the poorer LEC-dependent associative recognition memory detectable at pre-juvenile age is preceded by abnormal communication within LEC-HP-PFC

37 networks of neonatal mice mimicking the combined genetic and environmental etiology (GE) of disease. The prominent entorhinal drive to HP is weaker in GE mice as a result of sparser projections from LEC to CA1 and decreased efficiency of axonal terminals to activate the hippocampal circuits. In contrast, the direct entorhinal drive to PFC is not affected in GE

41 mice, yet the PFC is indirectly compromised, as target of the under-activated HP. Thus,

42 already at neonatal age, the entorhinal function gating prefrontal-hippocampal circuits is 43 impaired in a mouse model of disease. 


\section{INTRODUCTION}

The major burden of major psychiatric disorders, such as schizophrenia, is a lifelong cognitive disability ${ }^{1,2}$. Its devastating impact on the daily life is augmented by the fact that the available medication causes a weak, if any, improvement of cognitive deficits concerning attention, speed of processing, working and long-term memory, executive function, and social cognition, despite (almost) complete resolution of psychotic symptoms ${ }^{3}$. The pathophysiological substrate of these deficits has been identified to center on the prefrontalhippocampal network ${ }^{4,5}$. Abnormal prefrontal-hippocampal coupling during the tasks has been described in schizophrenia patients ${ }^{4,6}$. It relates not only to local alterations at microscopic and macroscopic scales in both areas $^{7}$ but also to connectivity dysfunction within large-scale networks ${ }^{8}$. In particular, the interactions between HP and entorhinal cortex, which tightly communicate with each other along reciprocal pathways ${ }^{9}$, have been tackled in clinical and neuropathological investigations ${ }^{10,} 11,12,13$. While some findings are still controversial, cytoarchitectural disorganization, cellular and synaptic deficits in layer 2 as well as aberrant axonal innervation have been detected in the entorhinal cortex of schizophrenia patients ${ }^{14,15}$. It has been hypothesized that they result from developmental disturbance of migration, differentiation and wiring of entorhinal circuits. However, the underlying mechanisms of these deficits and their contribution to the cognitive impairment are currently unknown.

Broader network dysconnectivity as underlying mechanism of cognitive deficits has been also identified in rodent models of mental illness ${ }^{16,17,18}$. While these models vary in their etiology, utility and validity, they provide precious insights into the neural basis of cognitive deficits that are difficult to obtain in humans ${ }^{19}$, especially when considering areas poorly investigated and less accessible, such as the entorhinal cortex. Lesion of the lateral entorhinal cortex (LEC) caused disease-characteristic altered mesolimbic dopaminergic transmission $^{20,21}$. Being a direct correlate of cognitive performance ${ }^{22}$, gamma oscillations within entorhinal-hippocampal circuits have been found to be disrupted in mouse models of disease $e^{23}$ 
Besides providing insights into the neurobiological substrate of disease, mouse models enable to test the neurodevelopmental origin of schizophrenia-related deficits. Recently, we showed that already during early postnatal development the neuronal activity and communication between limbic brain areas is profoundly compromised in a mouse model mimicking the genetic (knock-down of Disrupted-In-Schizophrenia $1\left(\right.$ Disc1 $\left.^{24}\right)$ and environmental (maternal immune activation (MIA)) etiology (GE) of mental illness ${ }^{25,26,27,28 .}$ Weaker communication between PFC and HP at neonatal age results from dysfunction of local pyramidal-interneuronal interactions as well as sparsification of hippocampal projections targeting the $\mathrm{PFC}^{28,29,30}$. At this age, LEC boosts the prefrontal-hippocampal circuits by projecting to $\mathrm{PFC}$ and $\mathrm{HP}^{31}$ and facilitating their oscillatory entrainment ${ }^{32}$. However, an in-depth characterization of the early network function and communication of LEC with PFC-HP pathway in disease models of mental illness is currently lacking.

To address this knowledge gap, here we combine in vivo electrophysiology and optogenetics with anatomical tracing and behavioral investigation to interrogate the developing entorhinal-hippocampal-prefrontal circuits in a mouse model mimicking the genetic and environmental etiology of mental illness. We show that in neonatal GE mice, sparser and less efficient projections from LEC cause weaker activation of HP and indirectly, of its target, the PFC.

\section{RESULTS}

\section{Pre-juvenile GE mice have poorer associative recognition memory}

One of the traits of cognitive impairment in schizophrenia is the poorer associative memory identified both in first episode and chronic patients ${ }^{33}$. This memory form requires precisely timed interactions within entorhinal-hippocampal circuits in humans ${ }^{34}$ and mice ${ }^{35}$. The strong lateral entorhinal input to HP provides non-spatial (contextual) information and facilitates the binding of information relating to objects, places, and contexts. To get first insights into the LEC function towards the end of development, we assessed the associative memory in GE when compared to control (CON) mice of pre-juvenile age (postnatal day $(P)$ 17-18). For this, 
two tasks were used: (i) novel object preference (distinct objects) (NOPd) task that involves an association between two different objects and (ii) object-location preference (OLP) task that involves an association between object and location (Fig. 1a, b). Mice performing these tasks showed an augmented neural activity in LEC mirrored by stronger cFos expression (supplementary fig. 1), suggesting that these two associative recognition tasks relates to LEC activation.

To test whether GE mice show poorer LEC-dependent associative recognition memory, we investigated CON $(n=20)$ and GE $(n=16)$ mice in NOPd and OLP tasks using a custom-designed arena. In line with our previous data ${ }^{36}$, we initiated the investigation after full maturation of sensory and motor abilities required for processing of novelty (P17). During the familiarization trial of the NOPd task, all mice spent equal time investigating the two objects placed in the arena (Fig. 1a). During the test trial, both CON mice and GE mice spent significantly longer time interacting with the novel object (CON: $146.66 \pm 9.42 \mathrm{~s}, p=$ 0.0009 , discrimination $=0.52 \pm 0.06$; GE: $115.89 \pm 7.06 \mathrm{~s}, p=0.04$, discrimination $=0.26 \pm$ 0.09) than with the familiar one (CON: $60.80 \pm 7.22 \mathrm{~s}$; GE: $93.03 \pm 12.93 \mathrm{~s})$ (Fig. 1a). However, GE mice had a poorer object discrimination when compared to CON mice (oneway ANOVA, $\mathrm{F}_{(1,38)}=6.95, p=0.012$ ). The behavioral impairment was detected only when the genetic deficit was paired with MIA and absent in one-hit G (Disc1 mutation alone) and one-hit $\mathrm{E}$ (MIA alone) mice that performed equally well as CON mice (supplementary fig. 2a). During the OLP task, the pre-juvenile mice had to associate object and location and distinguish, which object was placed in a newer location (Fig. 1b). In the test trial, the CON mice spent significantly longer time with the object with a newer location (new: $129.73 \pm 6.07$ s, old: $70.03 \pm 7.75 \mathrm{~s}, p=0.007$, discrimination $=0.34 \pm 0.07)$. GE mice equally investigated both objects (new: $102.94 \pm 6.06 \mathrm{~s}$, old: $74.42 \pm 12.16 \mathrm{~s}, p=0.09$, discrimination $=0.10 \pm$ 0.09). Correspondingly, the object discrimination was significantly lower in GE mice than in CON mice (one-way ANOVA, $\mathrm{F}_{(1,38)}=4.86, p=0.034$ ). One-hit $\mathrm{G}$ and one-hit $\mathrm{E}$ mice were also unable to recognize the object with a newer location (supplementary fig. 2b). 
Poor performance in NOPd and OLP tasks may result not only specifically from

dysfunction of entorhinal networks but also from poorer motor abilities and/or enhanced anxiety when interacting with the objects. To exclude these confounding effects, we analyzed the exploratory behavior of mice in the open field at P16. The motor abilities of both groups were comparable as reflected by the similar distance covered in the arena (GE: $2024.81 \pm 191.65 \mathrm{~cm}$ vs. CON: $2250.34 \pm 179.33 \mathrm{~cm}, p=0.24)$. All animals spent most of the time in the outer circle of the arena close to the walls and the distance covered in the inner circle was comparable between the groups (CON: $250 \pm 48 \mathrm{~cm}$; GE: $278 \pm 51 \mathrm{~cm}$ ). These data suggest that GE mice were not more anxious than CON mice.

Taken together, these results indicate that at pre-juvenile age the LEC-dependent associative recognition memory is impaired in GE mice.

The oscillatory entrainment of LEC within prefrontal-hippocampal networks is impaired in GE mice throughout development

The behavioral deficits might result from developmental disruption of LEC. To directly address this hypothesis, we investigated the oscillatory and firing activity of LEC and its embedding into PFC-HP networks. For this, we performed multi-site extracellular recordings of local field potential (LFP) and multiple-unit activity (MUA) from LEC, simultaneously with the CA1 area of intermediate/ventral HP (i/vCA1) and prelimbic subdivision (PL) of PFC of urethane-anesthetized P20-23 CON ( $\mathrm{P}=16)$, GE $(\mathrm{n}=11)$ mice in vivo (supplementary fig. 3). All investigated mice showed similar patterns of continuous network activity, which covered a broad frequencies spectrum and correspond to previously described sleep-like rhythms mimicked by urethane anesthesia ${ }^{37,38,39}$. While the oscillatory power in LEC as well as PFC and HP was comparable in CON and GE mice, the firing activity was significantly increased in both entorhinal layer $5 / 6$ (log firing rate, $1.65 \pm 0.18$ vs. $0.06 \pm 0.65, p=0.04$ ) and layer $2 / 3$ (log firing rate, $1.74 \pm 0.23$ vs. $-0.20 \pm 0.74, p=0.03$ ) as well as in stratum pyramidale of hippocampal CA1 area (log firing rate, $1.63 \pm 0.18$ vs. $-0.15 \pm 0.80, p=0.05$ ) in GE mice (supplementary fig. 3). The prefrontal firing activity was also increased in GE 
mice, yet below significance threshold (log firing rate, layer 5/6: $1.16 \pm 0.82$ vs. $0.28 \pm 0.63$, $p=0.37$; layer $2 / 3,1.40 \pm 0.63$ vs. $0.43 \pm 0.65, p=0.28)$.

In line with previous studies ${ }^{25,27,28}$, firing deficits at pre-juvenile age might reflect abnormal circuit wiring initiated at earlier stages of development. To test this hypothesis, we investigated the activity patterns within entorhinal-hippocampal-prefrontal networks in neonatal $(P 8-10)$ CON $(n=12)$ and GE $(n=10)$ mice (Fig. 2a). Extracellular recordings of LFP and MUA showed that discontinuous spindle-shaped oscillations with frequency components peaking in theta band $(4-12 \mathrm{~Hz})$ intermixed with irregular low amplitude betagamma band components $(12-30 \mathrm{~Hz})$ were the dominant pattern of entorhinal network activity of both groups of mice (Fig. 2a, supplementary fig. 4a). The discontinuous oscillatory events classified as spindle-bursts were superimposed on a slow rhythm $(2-4 \mathrm{~Hz})$ that continuously entrained the neonatal LEC (supplementary fig. 4a) and related to respiration ${ }^{40}$. The occurrence and duration of discontinuous oscillatory events $(4-30 \mathrm{~Hz})$ were comparable in CON and GE mice (supplementary fig. 4b). However, their power (4-30 Hz) was significantly smaller $\left(F_{(1,28)}=11.89, p=0.002\right.$, one-way ANOVA) in GE mice $(9.96 \pm 1.20)$ than CON mice $(23.45 \pm 3.39)$ (Fig. 2a). Given that single-hit $E$ and $G$ mice were indistinguishable in their activity patterns from CON mice (supplementary fig. 4c), single-hit models were not considered for the rest of investigations. The diminished network activity in LEC was accompanied, as previously reported ${ }^{25}$, by the dysfunction of network activity in both HP and PFC (Fig. 2b, c).

Next, we questioned whether the dampening of oscillatory activity in LEC, HP and PFC during early development related to communication deficits within the limbic networks. For this we firstly assessed the coupling by synchrony between LEC and PFC-HP pathway in neonatal CON $(n=14)$ and $G E(n=14)$ mice by calculating the coherence of oscillatory events and considering only the imaginary part that was not corrupted by volume conductance $^{41}$. A tight theta-beta band coupling of spindle-bursts between LEC and HP as well as between HP and PFC was detected in neonatal CON mice (Fig. 2d). In contrast, the imaginary coherence was significantly lower in GE mice (LEC-HP: $0.294 \pm 0.015$ vs. $0.256 \pm$ 
$0.010, F_{(1,26)}=5.18, p=0.03$, one-way ANOVA; HP-PFC: $0.345 \pm 0.013$ vs. $0.284 \pm 0.014$ $F_{(1,26)}=11.340, p=0.002$, one-way ANOVA) (Fig. 2d). The coherence between LEC and PFC was much higher than the coherence calculated for the shuffled data in both CON and GE mice, yet no frequency-specific coupling was detected (Fig. 2d). In a second step, to get better insights into the directionality of information flow within entorhinal-hippocampalprefrontal networks, we used the generalized partial directed coherence (gPDC), a measure that reflects the directionality of network interactions in different frequency bands. In CON mice, we confirmed the previously reported drive from HP to PFC as well as the stronger information flow from LEC to HP than from LEC to $\mathrm{PFC}^{32,42}$. The entorhinal drive to HP was significantly decreased in GE mice $(0.084 \pm 0.006$ vs. $0.112 \pm 0.006, p=0.0009$, one-way ANOVA), which, besides the previously reported local dysfunction in HP and PFC, might further contribute to the reduction of hippocampal drive to PFC $(0.069 \pm 0.004$ vs. $0.094 \pm$ 0.006, $p=0.002$, one-way ANOVA) (Fig. 2e). The weak drive entorhinal drive to PFC was comparable in CON and GE mice.

Taken together, these results uncover the functional pathways of communication within neonatal limbic circuits with the LEC boosting the hippocampal activity and, mainly via hippocampal activation, the PFC activity. Moreover, the data document the entorhinal dysfunction and abnormal coupling within limbic circuits of neonatal GE mice.

\section{Spatially distinct entorhinal projections to HP and PFC are sparser in neonatal GE mice}

One possible source of dysfunction within entorhinal-hippocampal-prefrontal circuits is the abnormal connectivity between these areas. We previously showed that already at the end of the first postnatal week hippocampal CA1 area strongly innervated the PFC, whereas no direct prefrontal projections targeted the HP. Moreover, we identified projections from LEC to HP as well as PFC in neonatal rats ${ }^{25,42}$. However, it is unknown, whether the same or distinct entorhinal populations innervate PFC and HP. To elucidate anatomical integration of LEC within the neonatal HP-PFC pathway, we injected the retrograde tracers CTB555 in HP 
and CTB488 in PFC of the same P7 mouse $(n=3)$ and monitored the projections after 3 days. CTB555 injection confined to i/vCA1 labeled cells mainly in layer $2 / 3$ of LEC (Fig. 3a). CTB488 injection confined to prelimbic-infralimbic subdivisions of the PFC labeled cells in the same layers, yet in a distinct, more superficial part; labeled neurons were detected also in $\mathrm{i} / \mathrm{vCA} 1$, confirming the previously described hippocampal projection to PFC (Fig. 3a). There was no overlap between the CTB555 and CTB488 labeled neurons in the superficial layers of LEC, indicating that the entorhinal HP- and PFC-projecting neurons had a distinct spatial organization.

The substrate of decreased functional coupling within entorhinal-hippocampalprefrontal networks in GE mice might be the sparser anatomical projections between the three areas in these mice. To test this hypothesis, we quantified the density of entorhinal HP- and PFC-projecting neurons in $\operatorname{CON}(n=4,3 \sim 4$ slices per mouse) and GE mice $(n=4$, 3 4 slices per mouse) (Fig. 3b, c). In line with the prominent entorhinal drive to HP and weaker drive to PFC in CON mice (Fig. 2e), the density of HP-projecting neurons was higher than the density of PFC-projecting neurons (Fig. 3b, c). GE mice showed a significantly ( $p=$ $0.00001)$ reduced density of HP-projecting neurons (1576.97 \pm 80.73$)$ but a similar $(p=0.92)$ density of PFC-projecting neurons $(774.66 \pm 97.42)$ when compared with CON mice $(2175.75 \pm 85.58,762.66 \pm 87.21)$ (Fig. 3b, c). To confirm the weaker innervation of HP by LEC axons in GE mice, we injected the anterograde tracer BDA in LEC at P7 and monitored the axonal terminals in HP of CON $(n=3)$ and GE mice $(n=3)$ at P10 (supplementary fig. 5). The high axonal density precluded the quantification of their length; instead, we monitored the intensity of the labeled terminals. GE mice showed a significantly reduced intensity of entorhinal axons in HP, suggesting that sparser projections link the LEC to hippocampal CA1 area in GE mice (supplementary fig. 5c). In contrast, very few BDA-labeled axonal terminals of entorhinal neurons were detected in PFC of both CON and GE mice.

Another source of dysfunction within entorhinal-hippocampal-prefrontal circuits in GE mice might represent the LEC neurons per se that due to abnormal properties are not able to provide the activation relayed to downstream areas. To test this hypothesis, we performed in 
vitro whole-cell patch-clamp recordings from entorhinal neurons that were either retrogradely labeled by CTB488 / Fluorogold (PFC-projecting neurons), or CTB555 (HP-projecting neurons). The passive membrane properties (RMP, $C_{m}, R_{\text {in }}, T m$ ) of PFC-projecting neurons as well as HP-projecting neurons were similar in P8-10 CON (27 neurons from 10 mice) and GE (12 neurons from 5 mice) mice (Table 1). All investigated neurons showed linear I-V relationships and their firing increased in response to depolarizing current injection. The active membrane properties (i.e. action potential (AP) threshold, AP amplitude, half-width, Rheobase, firing frequency) of both groups of entorhinal neurons did not differ between CON and GE mice (Table 1). These results suggest that circuit dysfunction of GE mice does not mainly relate to cellular abnormalities of entorhinal PFC-projecting and HP-projecting neurons.

\section{Weaker responsiveness of HP to optogenetic activation of LEC in neonatal GE mice}

To directly test the functional communication along axonal pathways within entorhinalhippocampal-prefrontal networks of CON and GE mice, we monitored the responsiveness of the three areas to the activation of LEC. For this, we selectively transfected pyramidal neurons in LEC of CON $(n=11)$ and GE $(n=13)$ mice with a highly efficient fast-kinetics double mutant ChR2E123T/T159C $(\mathrm{ET} / \mathrm{TC})^{43}$ and the red fluorescent protein mCherry by micro-injections performed at P1 (Fig. 4a, supplementary fig. 6).

First, we assessed the efficiency of light stimulation in evoking action potentials in entorhinal pyramidal neurons in vivo. Blue light pulses $\left(473 \mathrm{~nm}, 20-40 \mathrm{~mW} / \mathrm{mm}^{2}\right.$ ) at a frequency of $8 \mathrm{~Hz}$ led shortly $(<10 \mathrm{~ms})$ after the stimulus to precisely timed firing of transfected neurons in the LEC of both P8-10 CON and GE mice (Fig. 4b). The used light power did not cause local tissue heating that might interfere with neuronal spiking ${ }^{44,45}$. The efficiency of light stimulation in evoking entorhinal spikes was similar for both CON and GE groups (Fig. 4b). From the second pulse on, the firing of neurons from both groups gradually lost the precise timing to the stimulus and the response reliability, most likely due to the immaturity of entorhinal neurons unable to fire at the set frequency. Ramp light stimulation (3 
$\mathrm{s}, 473 \mathrm{~nm}, 20-40 \mathrm{~mW} / \mathrm{mm}^{2}$ ) induced theta-beta power peaks in the LEC of CON and GE mice (supplementary fig. 7). In line with the unweighted projections from the LEC to the two areas (Fig. 3), pulsed light stimulation in LEC led to rhythmic firing in HP, yet not in PFC (Fig. 4b). Quantification of the hippocampal firing probabilities upon stimulus revealed that CA1 neurons were reliably activated by light pulses in CON, yet not GE mice (Fig. 4b), most likely due to the reduced number of entorhinal HP-projecting neurons and the sparser entorhinal axonal terminals in HP.

Second, to decide whether LEC activation boosts information flow within entorhinalhippocampal-prefrontal networks, we investigated the synchrony of the three areas upon light stimulation in CON and GE mice. Ramp light stimulation augmented the $10-20 \mathrm{~Hz}$ coherence between LEC and HP $(0.14 \pm 0.05)$ but not between LEC and PFC $(-0.01 \pm 0.02)$ (Fig. 4c). Of note, frequency-specific boosting of HP through LEC activation caused an indirect augmentation of synchrony within prefrontal-hippocampal networks in CON mice $(0.19 \pm 0.06)$ (Fig. 4c). In GE mice, the stimulation-induced LEC-HP coherence increase was of lower magnitude $\left(0.04 \pm 0.03\right.$ vs. $\left.0.14 \pm 0.05, F_{(1,17)}=4.10, p=0.049\right)$ and consequently, not sufficient to augment the hippocampal-prefrontal synchrony (-0.03 \pm 0.04$)$ (Fig. 4c).

Taken together, these results indicate that LEC has a critical role for the activation of HP that on its turn boosts the entrainment of PFC. In contrast, the direct impact of entorhinal activity on PFC is low, if any. The hub function of LEC persists in GE mice, yet the LECdriven activation of HP is much weaker, being not further relayed to PFC.

\section{The function of entorhinal projections targeting the HP and PFC is selectively compromised in neonatal GE mice}

To experimentally backup the results above, we monitored the function of entorhinal axonal projections targeting either the PFC or the HP. In all investigated P8-10 mice LEC projected to the prelimbic and infralimbic sub-divisions of PFC, where it mainly targeted layer $5 / 6$ neurons (Fig. 5a). Light stimulation (3 ms, $473 \mathrm{~nm}, 8 \mathrm{~Hz}$ ) of terminals in PFC of ChR2transfected entorhinal neurons was performed simultaneously with extracellular LFP and 
290

291

292

293

294

295

296

297

298

299

300

301

302

303

304

305

306

307

308

309

310

311

312

MUA recordings in layer 5/6 of PL (Fig. 5b). Light activation of entorhinal terminals caused a pronounced short-delay ( $15 \mathrm{~ms}$ ) LFP depolarization and augmentation of neuronal firing in PFC of both CON and GE mice. The efficiency of light stimulation in evoking action potentials in layer 5/6 was low and comparable for CON and GE mice (0.19 \pm 0.01 vs. $0.18 \pm$ 0.02). Correspondingly, only 10 out $134(\sim 7.5 \%)$ prefrontal neurons in CON mice and 7 out $107(\sim 7.1 \%)$ in GE mice increased their firing upon light stimulation of entorhinal terminals (Fig. 5d).

Already at neonatal age, entorhinal projections target the HP (Fig. 3) and, in line with previous studies $^{46}$, accumulate in stratum lacunosum of CA1 area (Fig. 6a). The density of these projections significantly differs between CON and GE mice (Fig. 3, supplementary fig. 5). To test the function of entorhinal innervation of HP and whether the sparser projections in GE mice cause the network and neuronal deficits described above, we performed multi-site recordings of LFP and MUA in CA1 area during pulsed and ramp light stimulation of entorhinal terminals in HP (Fig. 6a). The field response evoked by light pulses (3 ms, $473 \mathrm{~nm}$, $8 \mathrm{~Hz})$ in HP had a fast $(\sim 15 \mathrm{~ms})$ onset in all investigated mice, yet a smaller amplitude $(23.37 \pm 3.91 \mu \mathrm{V}, p=0.040)$ in GE mice than CON mice $(50.63 \pm 11.79 \mu \mathrm{V})$ (Fig. 6b). Light stimulation of entorhinal terminals efficiently evoked hippocampal spikes of CON mice (probability $0.72 \pm 0.08$ ) (Fig. 6c), which was in line with the dense entorhinal axonal terminals (supplementary fig. 5). The firing probability upon stimulus was significantly $(p=$ $0.002)$ lower in the HP of GE mice $(0.32 \pm 0.05)$ (Fig. 6c). Not only the firing efficiency decreased but also the number of responsive hippocampal units was lower in GE mice (8 out $73, \sim 11 \%$ ) when compared to CON mice (22 out of $81, \sim 27 \%$ ) (Fig. 6d, e). These results indicate that the function of entorhinal projections in HP is impaired in GE mice, their efficiency to boost the hippocampal activity being decreased.

If the function of entorhinal projections to PFC but not to HP is largely intact in GE mice, the question arises, whether the weaker entorhinal drive to HP is still sufficient to entrain the neural activity in PFC. Light activation of entorhinal axonal terminals in HP (Fig. 7a) led to an increase of neuronal firing both in layer 5/6 (1.87 \pm 0.18$)$ and layer $2 / 3(1.70 \pm$ 
0.16) of PFC in CON mice (Fig. 7b, c). In contrast, the stimulation has a significantly weaker, if any, effect in the PFC of GE mice (layer 5/6, $1.50 \pm 0.12, p=0.04$; layer 2/3: $1.44 \pm 0.10, p$ $=0.04)$. Correspondingly, the prefrontal-hippocampal coupling augmented in CON mice during stimulating entorhinal terminals in HP (relative change $0.09 \pm 0.03$ ), yet not in GE mice (relative change, $-0.02 \pm 0.004, p=0.049$, Fig. $7 d$ ).

Taken together, these results uncover the pathways of functional wiring within entorhinal-hippocampal-prefrontal circuits and their disruption in GE mice. While the sparse entorhinal projections to PFC seem to be structurally and functionally normal in GE mice, the entorhinal-hippocampal communication is impaired, having indirect effects on the prefrontal activity.

\section{DISCUSSION}

Mouse models mimicking the dual etiology of schizophrenia, such as abnormal DISC1 function and immune challenge early in life reproduce the neuronal network and, to a certain amount, behavioral deficits of schizophrenia patients. The origin of these deficits has been hypothesized during development. Indeed, mice of an age corresponding to second-third gestational trimester in humans show prefrontal-hippocampal dysfunction ${ }^{25,} 27,28,29$, supporting the hypothesis of developmental miswiring in schizophrenia. The present results uncover novel mechanisms of miswiring in the neonatal brain and highlight the critical role of the LEC as gatekeeper of prefrontal-hippocampal circuits. We show that in mice mimicking the dual genetic-environmental etiology of schizophrenia (i) the patterns of oscillatory activity and coupling within LEC-HP-PFC networks are disrupted already at neonatal age, the entorhinal driving force on HP being particularly reduced, (ii) the substrate of abnormal LECHP coupling are sparser entorhinal axonal projections and their poorer efficiency to excite the HP, (iii) the disruption of entorhinal-hippocampal communication is sufficient to decrease the activation of PFC, whereas the direct entorhinal drive to PFC is largely unaffected, and (iv) the associative recognition memory that depends on entorhinal-hippocampal-prefrontal circuits and can be tested already at pre-juvenile age is poor when compared to CON mice. 
The entorhinal cortex represents a part of medial temporal lobe that is highly interconnected with the hippocampus and subcortical areas, such as the amygdala ${ }^{47}$. Related to its function and input-output connectivity, the entorhinal cortex has been divided into the medial entorhinal cortex (MEC) that is involved in spatial navigation and spatial memory ("Where") ${ }^{48}$ and LEC that codes context ("What") and temporal ("When") information ${ }^{49,50}$. In contrast to MEC, the LEC function has been less well dissected. It codes for object features and context-related locations, being critical for the performance in associative recognition memory of adults ${ }^{35}$. LEC is also involved in olfactory processing, as witnessed by lesions studies of LEC that led to olfactory anterograde amnesia ${ }^{51}$ but also facilitation of olfactory recognition ${ }^{52}$. Information transfer through LEC-HP synchrony is critical for olfactory associative learning ${ }^{53}$. Giving the LEC function and its tight embedding into large-scale circuits, it is not surprising that LEC came into the focus when investigating the pathophysiology of major psychiatric illnesses. Clinical, post-mortem and imaging studies identified structural and synaptic deficits in the entorhinal cortex of schizophrenia and bipolar disorder patients ${ }^{15,54,55,56}$. However, the mechanisms of these deficits remain largely unknown. It has been hypothesized that they result from abnormal development of LEC. LEC embedding into hippocampal-prefrontal circuits during development. Under normal conditions, the neonatal LEC acts as gatekeeper of HP and PFC, facilitating their activation. In line with the density of axonal projections, the direct entorhinal drive to HP is stronger than to PFC. On its turn, the hippocampal activation boosts the firing and oscillatory entrainment of PFC. By these means, two entorhinal pathways, directly and indirectly activate the PFC. They ensure the necessary level of neonatal excitation and oscillatory activation, which are mandatory for adult prefrontal-related behavior ${ }^{57}$. This knowledge gain might be instrumental for answering the question how non-sensory cortices, such as PFC, generate early patterns of oscillatory activity. Spontaneous activity from the periphery travels along axonal

371 projections via brainstem and thalamic nuclei and boosts the entrainment of developing 372 visual, barrel or auditory cortices in oscillatory rhythms that facilitate the emergence of 
373 characteristic functional topographies ${ }^{58,59}$. Such mechanisms are irrelevant for early 374 prefrontal oscillations; here, it seems that LEC drives the activation patterns. This mechanism is not fully decoupled from sensory inputs, since the LEC receives direct inputs from the olfactory bulb. The blind and deaf mouse pups that do not actively whisker at neonatal age have already adult-like olfactory abilities ${ }^{60}$. The olfactory bulb not only processes and forwards the odor information to the LEC, but also spontaneously generates patterns of early oscillatory activity that activate the LEC ${ }^{40,61}$. Therefore, at neonatal age, the entorhinal direct drive to HP as well as direct and indirect to PFC, is controlled by the olfactory system.

In mice mimicking the combined genetic and environmental etiology of disease, a prominent dysfunction within neonatal entorhinal-hippocampal-prefrontal networks has been identified. While the direct LEC-PFC communication was largely intact, the entorhinal drive to HP was weaker as result of sparser and reduced efficiency of axonal terminals to excite the hippocampal neurons. The effects of these deficits were detectable also in the downstream area, the PFC. Even if the passive and active membrane properties of entorhinal HP-projecting neurons were largely unaffected in GE mice, the neuronal function was impaired. The origin of this impairment is still unknown. One possible source might represent the dysfunction of local circuits in LEC. The cytoarchitecture of neonatal LEC was normal, yet subtle migration and differentiation deficits cannot be excluded as possible mechanisms, especially when considering that Disc1 gene represents an intracellular hub of developmental processes. Neurons in deep layers of LEC have abnormal passive and active properties (Rebecca Kringel, unpublished observations) that might perturb the intracortical entorhinal connectivity. Another source of entorhinal dysfunction might represent the weaker upstream input as result of olfactory deficits. Robust olfactory deficits have been identified in schizophrenia patients and at-risk youth ${ }^{62}$. The reduced size of the olfactory bulb and the resulting abnormal olfactory processing have been considered as a byproduct of an early developmental disturbance ${ }^{63}$. Thus, it can be hypothesized that the disturbance of LEC and downstream limbic circuitry involved in memory is, at least in part, the result of an early 
401

402

403

404

405

406

407

408

409

410

411

412

413

414

miswiring of olfactory system. Currently, investigations of olfactory processing of mouse models of disease are lacking. Their achievement might open new perspectives for mechanistic understanding of schizophrenia and in the end, for early diagnostic (i.e. biomarkers) and design of therapeutic strategies.

\section{MATERIALS AND METHODS}

Animal Models. All experiments were performed in compliance with the German laws and the guidelines of the European Community for the use of animals in research and were approved by the local ethical committee (015/17, 015/18). Timed-pregnant C57BL/6J mice from the animal facility of the University Medical Center Hamburg-Eppendorf were used. The day of vaginal plug detection was defined as gestational day $(G) 0.5$, whereas the day of birth was defined as postnatal day $(\mathrm{P})$ 0. Multisite extracellular recordings and behavioral testing were performed on pups of both sexes during neonatal development (i.e. P8-P10) as well as during pre-juvenile development (i.e. P16-P23). Heterozygous genetically engineered mutant DISC1 mice carrying a Disc1 allele (Disc1Tm1Kara) on a C57BL6/J background were used. Due to two termination codons and a premature polyadenylation site, the allele produces a truncated transcript ${ }^{64}$. Genotypes were determined using genomic DNA and following primer sequences: forward primer 5'-TAGCCACTCTCATTGTCAGC-3', reverse primer 5'-CCTCATCCCTTCCACTCAGC-3'. Mutant DISC1 mice were challenged by MIA, using the viral mimetic poly I:C $(5 \mathrm{mg} / \mathrm{kg})$ injected intravenously (i.v.) into the pregnant dams at gestational day G9.5. The resulting offspring mimicking the dual genetic-environmental etiology of mental disorders were classified as GE mice (DISC1 knock-down + MIA). The offspring of wild-type C57BL/6J dams injected at G9.5 with saline (0.9\%, i.v.) were classified as CON mice (control).

Electrophysiological recordings in vivo. For neonatal recordings in non-anesthetized state, $0.5 \%$ bupivacain / $1 \%$ lidocaine was locally applied on the neck muscles. For prejuvenile recordings under anesthesia, mice were injected intraperitoneally (i.p.) with urethane ( $1 \mathrm{mg} / \mathrm{g}$ body weight; Sigma-Aldrich, MO, USA) prior to surgery. For both age groups, under 
isoflurane anesthesia (induction: $5 \%$, maintenance: $2.5 \%$ ) the head of the pup was fixed into a stereotaxic apparatus using two plastic bars mounted on the nasal and occipital bones with dental cement. The bone above the PFC $(0.5 \mathrm{~mm}$ anterior to bregma, $0.1-0.5 \mathrm{~mm}$ right to the midline), hippocampus ( $3.5 \mathrm{~mm}$ posterior to bregma, $3.5 \mathrm{~mm}$ right to the midline), LEC (4 $\mathrm{mm}$ posterior to bregma, $6 \mathrm{~mm}$ right to the midline) was carefully removed by drilling a hole of $<0.5 \mathrm{~mm}$ in diameter. After a 10 min recovery period on a heating blanket, mouse was placed into the setup for electrophysiological recording. Throughout the surgery and recording session the mouse was positioned on a heating pad with the temperature kept at $37^{\circ} \mathrm{C}$.

A four-shank optoelectrode (NeuroNexus, MI, USA) containing $4 \times 4$ recording sites (0.4-0.8 M $\Omega$ impedance, $100 \mu \mathrm{m}$ spacing, $125 \mu \mathrm{m}$ intershank spacing) was inserted into the PL of PFC. A one-shank optoelectrode (NeuroNexus, MI, USA) containing 1x16 recordings sites (0.4-0.8 $\mathrm{M} \Omega$ impedance, $50 \mu \mathrm{m}$ spacing) was inserted into CA1 area. A one-shank optoelectrode (NeuroNexus, MI, USA) containing $1 \times 16$ recordings sites (0.4-0.8 M impedance, $100 \mu \mathrm{m}$ spacing) was inserted into LEC horizontally. An optical fiber ending 200 $\mu \mathrm{m}$ above the top recording site aligned with each recording shank. A silver wire was inserted into the cerebellum and served as ground and reference electrode. Extracellular signals were band-pass filtered $(0.1-9,000 \mathrm{~Hz})$ and digitized $(32 \mathrm{kHz})$ with a multichannel extracellular amplifier (Digital Lynx SX; Neuralynx, Bozeman, MO, USA) and the Cheetah acquisition software (Neuralynx). Spontaneous (i.e. not induced by light stimulation) activity was recorded for $20 \mathrm{~min}$ at the beginning of each recording session as baseline activity. The position of recording electrodes in the PL, CA1 area of $\mathrm{i} / \mathrm{VHP}$ and LEC was confirmed post mortem. Wide field fluorescence images were acquired to reconstruct the recording electrode position in brain slices of electrophysiologically investigated pups. Only pups with correct electrode position were considered for further analysis. In PL, the most medial shank was inserted to target layer $2 / 3$, whereas the most lateral shank was located into layer 5/6. For the analysis of hippocampal LFP, the recording site located in the pyramidal layer, where SPWs reverse ${ }^{65}$ was selected to minimize any non-stationary effects of large amplitude 
events. For the analysis of LEC LFP, the recording site that $700 \mu \mathrm{m}$ above the pyramidal layer of CA1 was selected.

Viral transfection in pyramidal neurons of LEC and light stimulation. Transfection of pyramidal neurons with a ChR2 derivate was achieved by injecting $0.1 \mu \mathrm{l}$ of the construct (AAV9-CaMKII-ChR2(E123T/T159C)-mCherry, at a titer $>1 \times 10^{13} \mathrm{vg} / \mathrm{mL}$, Addgene, Watertown, MA, USA) in LEC on the right hemisphere of $\mathrm{P0}-1$ pups. The pups were placed in a stereotactic apparatus and kept under anesthesia with isoflurane (induction: 5\%, maintenance: $2.5 \%$ ) for the entire procedure. A $10 \mathrm{~mm}$ incision of the skin on the head was performed with small scissors. The bone above the LEC was carefully removed using a syringe. The injection was achieved via a $10 \mu$ microsyringe pump controller. The injection speed $(0.05 \mu \mathrm{l} / \mathrm{min})$ was slow with the maintenance of the syringe in place for at least $8 \mathrm{~min}$. Pulsatile (laser on-off, pulse 3 ms-long, $8 \mathrm{~Hz}, 3$ s-long) or ramp (linearly increasing power, 3 s-long) light stimulations were performed with an arduino uno (Arduino, Italy) controlled diode laser (473 nm; Omicron, Austria). Laser power was adjusted to trigger neuronal spiking in response to $>25 \%$ of $3 \mathrm{~ms}$-long light pulses at $8 \mathrm{~Hz}$. Resulting light power was in the range of $20-40 \mathrm{~mW} / \mathrm{mm}^{2}$ at the fiber tip.

In vitro whole-cell patch-clamp recordings. Whole-cell patch-clamp recordings were performed from neurons identified by their location in the LEC and their projections to PFC (CTB488 / Fluorogold retrograde-labeled neurons) or HP (CTB555 retrograde-labeled neurons). All recordings were performed at room temperature. Recording electrodes (5$8 \mathrm{M} \Omega$ ) were filled with $\mathrm{K}$-gluconate based solution containing (in $\mathrm{mM}$ ):130 K-gluconate, 10 Hepes, 0.5 EGTA, 4Mg-ATP, 0.3Na-GTP, $8 \mathrm{NaCl}(285$ mosmol kg-1H2O, pH 7.4) and 0.5\% biocytin for post hoc morphological identification of recorded cells. Capacitance artefacts were minimized using the built-in circuitry of the patch-clamp amplifier (HEKA EPC 10, HEKA Elektronik, Germany). The signals were low-pass filtered at $10 \mathrm{kHz}$ and recorded online. All potentials were corrected for the liquid junction potential of the gluconate-based electrode solution, which, according to own measurement, was $-8.65 \mathrm{mV}$. The resting 
membrane potential (RMP) was measured immediately after obtaining the whole-cell configuration. For the determination of input resistance (Rin), membrane time constant (Tm) and membrane capacitance $(\mathrm{Cm})$, hyper-polarizing current pulses $(-60 \mathrm{pA})$ of $600 \mathrm{~ms}$ in duration were applied from the resting membrane potential. Firing frequency was assessed at a depolarizing current pulse of $100 \mathrm{pA}$ at the same length of $600 \mathrm{~ms}$. Analysis was performed offline using custom-written scripts in the MATLAB environment.

Behavioral protocols. The exploratory behavior and recognition memory of CON and GE mice were tested at pre-juvenile age using previously established experimental protocols ${ }^{66}$. Briefly, all behavioral tests were conducted in a custom-made circular white arena, the size of which (D: $34 \mathrm{~cm}, \mathrm{H}: 30 \mathrm{~cm}$ ) maximized exploratory behavior, while minimizing incidental contact with testing objects ${ }^{67}$. The objects used for testing of associative recognition were six differently shaped, textured and colored, easy to clean items that were provided with magnets to fix them to the bottom of the arena. Object sizes $(\mathrm{H}: 3 \mathrm{~cm}$, diameter: $1.5-3 \mathrm{~cm})$ were smaller than twice the size of the mouse and did not resemble living stimuli (no eye spots, predator shape). The objects were positioned at $10 \mathrm{~cm}$ from the borders and $8 \mathrm{~cm}$ from the center of the arena. After every trial the objects and arena were cleaned with $0.1 \%$ acetic acid to remove all odors. A black and white CCD camera (VIDEOR TECHNICAL E. Hartig GmbH, Roedermark, Germany) was mounted $100 \mathrm{~cm}$ above the arena and connected to a $\mathrm{PC}$ via $\mathrm{PCl}$ interface serving as frame grabber for video tracking software (Video Mot2 software, TSE Systems GmbH, Bad Homburg, Germany).

Exploratory behavior in the open field. Pre-juvenile mice (P16) were allowed to freely explore the testing arena for $10 \mathrm{~min}$. Additionally, the floor area of the arena was digitally subdivided in 8 zones ( 4 center zones and 4 border zones) using the zone monitor mode of the VideoMot 2 analysis software (VideoMot 2, TSE Systems $\mathrm{GmbH}$ ). The time spent by pups in center and border zones, as well as the running distance and velocity was quantified.

Novel object preference (distinct objects) (NOPd) and object-location preference (OLP) tasks. All protocols for assessing associative recognition memory in P17 (NOPd) and P18 
510 (OLP) mice consisted of familiarization and test trials. During the familiarization trial each

511 mouse was placed into the arena containing two different objects and released with the back

512 to the objects. After $10 \mathrm{~min}$ of free exploration of objects the mouse was returned to a 513 temporary holding cage. Subsequently, the test trial was performed after a delay of 5 min 514 post-familiarization. In NOPd task, the mice were allowed to investigate one familiar and one novel object with a different shape and texture for $5 \mathrm{~min}$. The nature of this test is similar to the novel object preference test, except that the test trial involves an association between two different objects (an association of object-object). In OLP task, the mice were allowed to investigate one familiar and a copy of the old object that was previously presented for 5 min. This test examines whether animals recognize the location that was once occupied by a particular object (an association of object-location). Object interaction during the first 4 min was analyzed and compared between the groups. All trials were video-tracked and the analysis was performed using the Video Mot2 analysis software. The object recognition module of the software was used and a 3-point tracking method identified the head, the rear end and the center of gravity of the mouse. Digitally, a circular zone of $1.5 \mathrm{~cm}$ was created around each object and every entry of the head point into this area was considered as object interaction. Climbing or sitting on the object, mirrored by the presence of both head and center of gravity points within the circular zone, were not counted as interactions.

The object discrimination was computed for NOPd and OLP as (time at object2- time at object1) / (time at object1+ time at object2) (Fig. 1).

OLP task. P16 CON mice were randomly divided into 2 groups ( $\mathrm{n}=4$ mice / group). The mice were allowed to freely explore the arena containing two different objects for 10 mins. This familiarization process continued for 3 days with 2 trials per day. On the third day (P18), 5 mins after the last familiarization trial, 3 mice from one group were signed to perform the test trial (5 mins) of NOPd task, whereas one mouse to perform the familiarization trial. 
537

538

539

540

541

542

543

544

545

546

547

548

549

550

551

552

553

554

555

556

557

task and one mouse to perform the familiarization trial. The mice were perfused $\sim 90$ min after the last behavioral trial.

Retrograde tracing. For retrograde tracing, P7 mice received retrograde tracer CTB555 (Cholera Toxin Subunit B, Alexa Fluor 455 Conjugate) injections into HP $(0.7 \mathrm{~mm}$ anterior from the lambda, $2.4 \mathrm{~mm}$ from midline, $1.6 \mathrm{~mm}$ depth), and CTB488 (Cholera Toxin Subunit $\mathrm{B}$, Alexa Fluor 488 Conjugate) injections into PFC $(0.7 \mathrm{~mm}$ anterior from to bregma, $0.1 \mathrm{~mm}$ from midline, $1.9 \mathrm{~mm}$ depth). The pups were placed in a stereotactic apparatus and kept under anesthesia with isoflurane (induction: 5\%, maintenance: $2.5 \%$ ) for the entire procedure. A $10 \mathrm{~mm}$ incision of the skin on the head was performed with small scissors. The bone above the HP and PFC was carefully removed using a syringe. A total volume of $0.1 \mu \mathrm{l}$ of CTB (2.5\% in PBS) was delivered via a $10 \mu \mathrm{l}$ microsyringe pump controller into PFC or HP. The slow injection speed $(0.05 \mu \mathrm{l} / \mathrm{min})$ and the maintenance of the syringe in place for at least 8 min ensured an optimal diffusion of the tracer. The pups were perfused at P10.

Anterograde tracing. Two anterograde tracers were used. First, to quantify the axonal terminals from LEC to HP, anterograde tracer biotinylated dextran amine (BDA) (Thermo Fisher Scientific, USA) was used. Mice were injected at P7 with BDA unilaterally into LEC using iontophoresis. The bone above LEC was carefully removed using a syringe. A glass capillary $(\sim 30 \mu \mathrm{m}$ tip diameter) was filled with $\sim 1 \mu \mathrm{L}$ of $5 \%$ BDA diluted in $0.125 \mathrm{M}$ phosphate buffer by capillary forces, and a silver wire was inserted such that it was in contact with the BDA solution. The positive pole of the iontophoresis device was attached to the silver wire, the negative one was attached to the skin of the neck. Iontophoretically injection by applying anodal current to the pipette (6s on/off current pulses of $6 \mu \mathrm{A}$ ) was done for $10 \mathrm{~min}$. Following injection, the pipette was left in place for at least 5 min and then slowly retracted. The scalp was closed by application of tissue adhesive glue and the pups were left on a heating pad for 10-15 min to fully recover before they were given back to the mother. The pups were perfused at P10. Second, to locate the innerved neurons in PFC by LEC, anterograde trans-synaptic tracer wheat germ agglutinin (WGA) (Thermo Fisher 
564

565

566

567

568

569

570

571

572

573

574

575

576

577

578

579

580

581

582

583

584

585

586

587

588

589

590

Scientific, USA) was used. Mice were injected at P8 with WGA unilaterally into LEC. A total volume of $0.1 \mu \mathrm{l}$ of WGA (2.5\% in PBS) was delivered via a $10 \mu \mathrm{l}$ microsyringe pump controller. The slow injection speed $(0.05 \mu \mathrm{l} / \mathrm{min})$ and the maintenance of the syringe in place for at least 8 min ensured an optimal diffusion of the tracer. 40 hours after the injection, the pups were perfused.

Histology and staining protocols. Histological procedures were performed as previously described $^{26,27}$. Briefly, P8-10 and P18-23 mice were anesthetized with $10 \%$ ketamine (aniMedica) / $2 \%$ xylazine (WDT) in $0.9 \% \mathrm{NaCl}$ solution $(10 \mu \mathrm{g} / \mathrm{g}$ body weight, i.p.) and transcardially perfused with Histofix (Carl Roth) containing 4\% paraformaldehyde. Brains were postfixed in Histofix for $24 \mathrm{~h}$ and sectioned coronally at $50 \mathrm{~mm}$ (immunohistochemistry) or $100 \mathrm{~mm}$ (quantification for CTB labeled neurons). Free-floating slices were permeabilized and blocked with PBS containing $0.8 \%$ Triton X 100 (Sigma-Aldrich, MO, USA), 5\% normal bovine serum (Jackson Immuno Research, PA, USA) and $0.05 \%$ sodium azide. For cFos staining, slices were incubated with mouse monoclonal Alexa Fluor-555 conjugated antibody against cFos (1:200, MAB377X, Merck Millipore, MA, USA), followed by $2 \mathrm{~h}$ incubation with Alexa Fluor-488 goat anti-rabbit IgG secondary antibody (1:500, A11008, Merck Millipore, MA). For BDA staining, slices were incubated with streptavidin (Сy3, 1:500, ThermoFisher). For WGA staining, slices were incubated with goat anti-WGA IgG (1:500), followed by $2 \mathrm{~h}$ incubation with Alexa Fluor-488 goat anti-rabbit lgG secondary antibody (1:500, A11008, Merck Millipore, MA). Slices were transferred to glass slides and covered with Fluoromount (Sigma-Aldrich, MO, USA). Wide-field fluorescence images were acquired to reconstruct the recording electrode position.

Quantification of cFos, CTB488, CTB555, WGA labeled neurons. All quantifications were carried out blind to the experimental condition. Using a light microscope, photographs of the relevant areas (LEC for cFos, CTB488, CTB555; PFC for WGA) were taken with a consistent light level (Olympus FX-100). 3 4 slices per animal were used. Images were processed using ImageJ software. The number of cFos positive neurons, CTB488 positive 
591

592

neurons, CTB555 positive neurons and WGA positive neurons were counted manually in the interested regions.

Quantification of BDA-labeled axonal terminals. High magnification images were acquired by confocal microscopy (DM IRBE, Leica Microsystems, Zeiss LSN700) from stratum lacunosum-moleculare of CA1 to quantify LEC axonal terminals labeled by BDA. Microscopic stacks were acquired as $2048 \times 2048$ pixel images (pixel size, 78 nm; Z-step, $500 \mathrm{~nm}$ ). All images were similarly processed and analyzed using ImageJ software.

Data Analysis. Data were imported and analyzed offline using custom-written tools in Matlab software version 7.7 (Mathworks). The data were processed as following: (i) bandpass filtered $(500-5000 \mathrm{~Hz})$ to detect MUA as negative deflections exceeding five times the standard deviation of the filtered signals and (ii) low-pass filtered $(<1500 \mathrm{~Hz})$ using a third order Butterworth filter before downsampling to $1000 \mathrm{~Hz}$ to analyze the LFP. All filtering procedures were performed in a phase-preserving manner. The position of Dil-stained recording electrodes in PL (most medial shank confined to layer 2/3, most temporal shank confined to layer 5/6), CA1 and LEC was confirmed post-mortem by histological evaluation. Additionally, electrophysiological features (i.e. reversal of LFP and high MUA frequency over stratum pyramidale of CA1) were used for confirmation of the exact recording position in HP.

Detection of neonatal oscillatory activity. Discontinuous oscillatory events were dected using a previously developed unsupervised algorithm ${ }^{68}$ and confirmed by visual inspection. Briefly, deflections of the root-mean-square of band-pass $(3-100 \mathrm{~Hz})$ filtered signals exceeding a variance-depending threshold were assigned as network oscillations. The threshold was determined by a Gaussian fit to the values ranging from 0 to the global maximum of the root-mean-square histogram. Only oscillatory events $>1 \mathrm{~s}$ were considered for further analysis. Time-frequency plots were calculated by transforming the data using the Morlet continuous wavelet.

Power spectral density. For power spectral density analysis, 1 s-long window of network oscillations were concatenated and the power was calculated using Welch's method with 
618

non-overlapping windows. For optical stimulation, we compared the average power during the $1.5 \mathrm{~s}$-long time window preceding the stimulation to the last $1.5 \mathrm{~s}$-long time window of light-evoked activity.

Single unit activity (SUA). SUA was detected and clustered using klusta (Rossant et al., 2016) and manually curated using phy (https://github.com/cortex-lab/phy). Data were imported and analyzed using custom-written tools in the MATLAB. Spikes occurring in a 20 ms-long time window after the start of a light pulse were considered to be light-evoked. Stimulation efficacy was calculated as the probability of at least one spike occurring in this period.

Firing rate. The firing rate was computed by dividing the total number of spikes by the duration of the analyzed time window.

Spectral coherence. Coherence was calculated using the coherence method. Briefly, the coherence was calculated (using the functions cpsd.m and pwelch.m) by cross-spectral density between the two signals and normalized by the power spectral density of each. The computation of the coherence $\mathrm{C}$ over frequency $(f)$ for the power spectral density $\mathrm{P}$ of signal $\mathrm{X}$ and $\mathrm{Y}$ was performed according to the formula:

$$
C_{X Y}(f)=\left|\left(\frac{P_{X Y}(f)}{\sqrt{P_{X X}(f) P_{Y Y}(f)}}\right)\right|
$$

Directionality methods. To investigate the directionality of functional connectivity between PFC and HP, gPDC was used. gPDC is based on linear Granger causality measure in the frequency domain. The method attempts to describe the causal relationship between multivariate time series based on the decomposition of multivariate partial coherence computed from multivariate autoregressive models. The LFP signal was divided into 1s-long segments containing the oscillatory activity. After de-noising using Matlab wavelet toolbox, gPDC was calculated using a previously described algorithm ${ }^{69,70}$. 
641 Estimation of light propagation. The spatial pattern of light propagation in vivo was 642 estimated using a previously developed model ${ }^{45}$ based on Monte Carlo simulation (probe 643 parameters: light fiber diameter: $50 \mu \mathrm{m}$, numerical aperture: 0.22, light parameters: $594 \mathrm{~nm}$, $6440.6 \mathrm{~mW})$.

645 Statistical analysis. Statistical analyses were performed in Matlab environment. Significant 646 differences were detected by paired t-test or one-way ANOVA. Investigators were blinded to 647 the group allocation when the quantifications of cFos expression, CTB488 and CTB555 648 positive neurons in LEC, and WGA positive neurons in PFC were performed. Data are 649 presented as mean \pm sem. Significance levels of $p<0.05\left(^{*}\right), p<0.01\left(^{* *}\right)$ or $p<0.001\left(^{* * *}\right)$ 650 were tested. Statistical parameters can be found in the main text.

\section{Code availability}

652 All the codes used in the current study are available from the corresponding author upon 653 request.

\section{Data availability}

655 The data supporting the findings of this study is available with the article and its 656 Supplementary Information file, or is available from the corresponding author upon request. 
657

658

659

660

661

662

663

664

665

666

667

668

669

670

671

672

673

674

675

676

677

678

679

680

681

682

683

684

685

686

687

688

689

690

\section{References}

1. van Os J, Kenis G, Rutten BPF. The environment and schizophrenia. Nature 468, 203-212 (2010).

2. Howes OD, Murray RM. Schizophrenia: an integrated sociodevelopmental-cognitive model. Lancet 383, 1677-1687 (2014).

3. Goldberg AD, Allis CD, Bernstein E. Epigenetics: A Landscape Takes Shape. Cell 128, 635-638 (2007).

4. Meyer-Lindenberg A, et al. Neural correlates of genetically abnormal social cognition in Williams syndrome. Nat Neurosci 8, 991-993 (2005).

5. Bähner F, Meyer-Lindenberg A. Hippocampal-prefrontal connectivity as a translational phenotype for schizophrenia. Eur Neuropsychopharmacol 27, 93-106 (2017).

6. Rasetti R, Sambataro F, Chen Q, Callicott JH, Mattay VS, Weinberger DR. Altered Cortical Network Dynamics: A Potential Intermediate Phenotype for Schizophrenia and Association With ZNF804A. Arch Gen Psychiatry 68, 1207-1217 (2011).

7. Meyer-Lindenberg A. From maps to mechanisms through neuroimaging of schizophrenia. Nature 468, 194-202 (2010).

8. Kupferschmidt DA, Gordon JA. The dynamics of disordered dialogue: Prefrontal, hippocampal and thalamic miscommunication underlying working memory deficits in schizophrenia. BNA 2, 2398212818771821 (2018).

9. Nilssen ES, Doan TP, Nigro MJ, Ohara S, Witter MP. Neurons and networks in the entorhinal cortex: A reappraisal of the lateral and medial entorhinal subdivisions mediating parallel cortical pathways. Hippocampus 29, 1238-1254 (2019).

10. Arnold PJ. Dimensional Standardization and Production Scale in Mesoamerican Ceramics. Lat Am Antiq 2, 363-370 (1991).

11. Arnold SE. Cellular and Molecular Neuropathology of the Parahippocampal Region in Schizophrenia. Ann N Y Acad Sci 911, 275-292 (2000).

12. Baiano $\mathrm{M}$, et al. Decreased entorhinal cortex volumes in schizophrenia. Schizophr Res 102, 171-180 (2008).

13. Akil M, Lewis DA. Cytoarchitecture of the entorhinal cortex in schizophrenia. Am J Psychiatry 154, 1010-1012 (1997).

14. Hemby SE, Ginsberg SD, Brunk B, Arnold SE, Trojanowski JQ, Eberwine JH. Gene Expression Profile for Schizophrenia: Discrete Neuron Transcription Patterns in the Entorhinal Cortex. Arch Gen Psychiatry 59, 631-640 (2002). 
15. Falkai P, Schneider-Axmann T, Honer WG. Entorhinal cortex pre-alpha cell clusters in schizophrenia: quantitative evidence of a developmental abnormality. Biol Psychiatry 47, 937-943 (2000).

16. Bolkan SS, et al. Thalamic projections sustain prefrontal activity during working memory maintenance. Nat Neurosci 20, 987-996 (2017).

17. Parnaudeau S, et al. Inhibition of Mediodorsal Thalamus Disrupts Thalamofrontal Connectivity and Cognition. Neuron 77, 1151-1162 (2013).

18. Chun S, et al. Specific disruption of thalamic inputs to the auditory cortex in schizophrenia models. Science 344, 1178-1182 (2014).

19. Insel TR. Rethinking schizophrenia. Nature 468, 187-193 (2010).

20. Sumiyoshi T, et al. Enhanced locomotor activity in rats with excitotoxic lesions of the entorhinal cortex, a neurodevelopmental animal model of schizophrenia: Behavioral and in vivo microdialysis studies. Neurosci Lett 364, 124-129 (2004).

21. Meyer F, Peterschmitt $Y$, Louilot A. Postnatal functional inactivation of the entorhinal cortex or ventral subiculum has different consequences for latent inhibition-related striatal dopaminergic responses in adult rats. Eur J Neurosci 29, 2035-2048 (2009).

22. Fell $\mathrm{J}$, et al. Human memory formation is accompanied by rhinal-hippocampal coupling and decoupling. Nat Neurosci 4, 1259-1264 (2001).

23. Cunningham MO, et al. Region-Specific Reduction in Entorhinal Gamma Oscillations and Parvalbumin-Immunoreactive Neurons in Animal Models of Psychiatric Illness. $J$ Neurosci 26, 2767 (2006).

24. Brandon NJ, Sawa A. Linking neurodevelopmental and synaptic theories of mental illness through DISC1. Nat Rev Neurosci 12, 707-722 (2011).

25. Hartung $\mathrm{H}$, et al. From Shortage to Surge: A Developmental Switch in HippocampalPrefrontal Coupling in a Gene-Environment Model of Neuropsychiatric Disorders. Cereb Cortex 26, 4265-4281 (2016).

26. Oberlander VC, Xu X, Chini M, Hanganu-Opatz IL. Developmental dysfunction of prefrontal-hippocampal networks in mouse models of mental illness. Eur $\mathrm{J}$ Neurosci 50, 3072-3084 (2019).

27. Xu X, Chini M, Bitzenhofer SH, Hanganu-Opatz IL. Transient Knock-Down of Prefrontal DISC1 in Immune-Challenged Mice Causes Abnormal Long-Range Coupling and Cognitive Dysfunction throughout Development. J Neurosci 39, 1222 (2019).

28. Xu X, Song L, Hanganu-Opatz IL. Knock-Down of Hippocampal DISC1 in ImmuneChallenged Mice Impairs the Prefrontal-Hippocampal Coupling and the Cognitive Performance Throughout Development. Cereb Cortex 31, 1240-1258 (2021). 
29. Chini M, et al. Resolving and Rescuing Developmental Miswiring in a Mouse Model of Cognitive Impairment. Neuron 105, 60-74.e67 (2020).

30. Comer AL, et al. Increased expression of schizophrenia-associated gene C4 leads to hypoconnectivity of prefrontal cortex and reduced social interaction. PLOS Biol 18, e3000604 (2020).

31. Jones BF, Witter MP. Cingulate cortex projections to the parahippocampal region and hippocampal formation in the rat. Hippocampus 17, 957-976 (2007).

32. Hartung $\mathrm{H}$, Brockmann MD, Pöschel B, De Feo V, Hanganu-Opatz IL. Thalamic and Entorhinal Network Activity Differently Modulates the Functional Development of Prefrontal-Hippocampal Interactions. J Neurosci 36, 3676 (2016).

33. Mathew I, et al. Medial Temporal Lobe Structures and Hippocampal Subfields in Psychotic Disorders: Findings From the Bipolar-Schizophrenia Network on Intermediate Phenotypes (B-SNIP) Study. JAMA Psychiatry 71, 769-777 (2014).

34. Staresina BP, Reber TP, Niediek J, Boström J, Elger CE, Mormann F. Recollection in the human hippocampal-entorhinal cell circuitry. Nat Commun 10, 1503 (2019).

35. Wilson DIG, Watanabe S, Milner H, Ainge JA. Lateral entorhinal cortex is necessary for associative but not nonassociative recognition memory. Hippocampus 23, 1280 1290 (2013).

36. Krüger H-S, Brockmann MD, Salamon J, Ittrich H, Hanganu-Opatz IL. Neonatal hippocampal lesion alters the functional maturation of the prefrontal cortex and the early cognitive development in pre-juvenile rats. Neurobiol Learn Mem 97, 470-481 (2012).

37. Wolansky T, Clement EA, Peters SR, Palczak MA, Dickson CT. Hippocampal Slow Oscillation: A Novel EEG State and Its Coordination with Ongoing Neocortical Activity. J Neurosci 26, 6213 (2006).

38. Clement EA, Richard A, Thwaites M, Ailon J, Peters S, Dickson CT. Cyclic and Sleep-Like Spontaneous Alternations of Brain State Under Urethane Anaesthesia. PLOS ONE 3, e2004 (2008).

39. Pagliardini S, Gosgnach S, Dickson CT. Spontaneous Sleep-Like Brain State Alternations and Breathing Characteristics in Urethane Anesthetized Mice. PLOS ONE 8, e70411 (2013).

40. Gretenkord S, et al. Coordinated electrical activity in the olfactory bulb gates the oscillatory entrainment of entorhinal networks in neonatal mice. PLoS Biol 17, e2006994 (2019).

41. Nolte G, Bai O, Wheaton L, Mari Z, Vorbach S, Hallett M. Identifying true brain interaction from EEG data using the imaginary part of coherency. Clin Neurophysiol 115, 2292-2307 (2004). 
42. Brockmann Marco D, Pöschel B, Cichon N, Hanganu-Opatz Ileana L. Coupled Oscillations Mediate Directed Interactions between Prefrontal Cortex and Hippocampus of the Neonatal Rat. Neuron 71, 332-347 (2011).

43. Berndt $A$, et al. High-efficiency channelrhodopsins for fast neuronal stimulation at low light levels. PNAS 108, 7595-7600 (2011).

44. Bitzenhofer SH, Ahlbeck J, Hanganu-Opatz IL. Methodological Approach for Optogenetic Manipulation of Neonatal Neuronal Networks. Front Cell Neurosci 11, 239 (2017).

45. Stujenske JM, Spellman T, Gordon JA. Modeling the Spatiotemporal Dynamics of Light and Heat Propagation for In Vivo Optogenetics. Cell Rep 12, 525-534 (2015).

46. Kitamura T, Macdonald CJ, Tonegawa S. Entorhinal-hippocampal neuronal circuits bridge temporally discontiguous events. Learn Mem 22, 438-443 (2015).

47. Witter MP, Wouterlood FG, Naber PA, Van Haeften T. Anatomical Organization of the Parahippocampal-Hippocampal Network. Ann N Y Acad Sci 911, 1-24 (2000).

48. Moser El, Moser M-B, McNaughton BL. Spatial representation in the hippocampal formation: a history. Nat Neurosci 20, 1448-1464 (2017).

49. Tsao A, et al. Integrating time from experience in the lateral entorhinal cortex. Nature 561, 57-62 (2018).

50. Wang $\mathrm{C}$, et al. Egocentric coding of external items in the lateral entorhinal cortex. Science 362, 945-949 (2018).

51. Staubli U, Fraser D, Kessler M, Lynch G. Studies on retrograde and anterograde amnesia of olfactory memory after denervation of the hippocampus by entorhinal cortex lesions. Behav Neural Biol 46, 432-444 (1986).

52. Wirth S, Ferry B, Di Scala G. Facilitation of olfactory recognition by lateral entorhinal cortex lesion in rats. Behav Brain Res 91, 49-59 (1998).

53. Igarashi KM, Lu L, Colgin LL, Moser M-B, Moser El. Coordination of entorhinalhippocampal ensemble activity during associative learning. Nature 510, 143-147 (2014).

54. Bachus SE, Hyde TM, Herman MM, Egan MF, Kleinman JE. Abnormal cholecystokinin mRNA levels in entorhinal cortex of schizophrenics. J Psychiatr Res 31, 233-256 (1997).

55. Benes FM, Berretta S. GABAergic Interneurons: Implications for Understanding Schizophrenia and Bipolar Disorder. Neuropsychopharmacology 25, 1-27 (2001).

56. Pantazopoulos H, Lange N, Baldessarini RJ, Berretta S. Parvalbumin Neurons in the Entorhinal Cortex of Subjects Diagnosed With Bipolar Disorder or Schizophrenia. Biol Psychiatry 61, 640-652 (2007). 
800

801

802

803

804

805

806

807

808

809

810

811

812

813

814

815

816

817

818

819

820

821

822

823

824

825

826

827

828

829

830

831

832

833

834

57. Bitzenhofer SH, Pöpplau JA, Chini M, Marquardt A, Hanganu-Opatz IL. Transient developmental increase of prefrontal activity alters network maturation and causes cognitive dysfunction in adult mice. Neuron, in press, (2020).

58. Khazipov R, Sirota A, Leinekugel X, Holmes GL, Ben-Ari Y, Buzsáki G. Early motor activity drives spindle bursts in the developing somatosensory cortex. Nature 432 , 758-761 (2004).

59. Hanganu IL, Ben-Ari Y, Khazipov R. Retinal Waves Trigger Spindle Bursts in the Neonatal Rat Visual Cortex. J Neurosci 26, 6728 (2006).

60. Welker W. Analysis of Sniffing of the Albino Rat. Behaviour 22, 223-224 (1964).

61. Kostka JK, Gretenkord S, Spehr M, Hanganu-Opatz IL. Bursting mitral cells time the oscillatory coupling between olfactory bulb and entorhinal networks in neonatal mice. J Physiol 598, 5753-5769 (2020).

62. Moberg PJ, et al. Meta-Analysis of Olfactory Function in Schizophrenia, First-Degree Family Members, and Youths At-Risk for Psychosis. Schizophr Bull 40, 50-59 (2014).

63. Turetsky BI, et al. Structural anomalies of the peripheral olfactory system in psychosis high-risk subjects. Schizophr Res 195, 197-205 (2018).

64. Kvajo $M$, et al. A mutation in mouse Disc1 that models a schizophrenia risk allele leads to specific alterations in neuronal architecture and cognition. PNAS 105, 70767081 (2008).

65. Bitzenhofer SH, Hanganu-Opatz IL. Oscillatory coupling within neonatal prefrontalhippocampal networks is independent of selective removal of GABAergic neurons in the hippocampus. Neuropharmacology 77, 57-67 (2014).

66. Kruger HS, Brockmann MD, Salamon J, Ittrich H, Hanganu-Opatz IL. Neonatal hippocampal lesion alters the functional maturation of the prefrontal cortex and the early cognitive development in pre-juvenile rats. Neurobiol Learn Mem 97, 470-481 (2012).

67. Heyser CJ, Ferris JS. Object exploration in the developing rat: methodological considerations. Dev Psychobiol 55, 373-381 (2013).

68. Cichon NB, Denker M, Grun S, Hanganu-Opatz IL. Unsupervised classification of neocortical activity patterns in neonatal and pre-juvenile rodents. Front Neural Circuits 8, 50 (2014).

69. Baccala LA, Sameshima K. Partial directed coherence: a new concept in neural structure determination. Biol Cybern 84, 463-474 (2001).

70. Baccala LA, Sameshima K, Takahashi DY. Generalized Partial Directed Coherence. In: 2007 15th International Conference on Digital Signal Processing (2007). 
835

836

837

838

839

840

841

842

843

844

845

846

847

\section{Acknowledgements}

We thank Dr. Joseph Gogos for providing the DISC1 mice. We also thank A. Marquardt, C. Tietze, A. Dahlmann, and P. Putthoff for excellent technical assistance. This work was funded by grants from the European Research Council (ERC-2015-CoG 681577 to I.L.H.-O.) and the German Research Foundation (SPP 1665, SFB 936 B5 to I.L.H.-O).

\section{Author contributions}

I.L.H.-O., X.X. designed the experiments, X.X., L.S., and R.K carried out the experiments, X.X. analyzed the data, I.L.H.-O. and X.X. interpreted the data and wrote the paper. All authors discussed and commented on the manuscript.

\section{Competing interests}

The authors declare no competing financial interests. 
a
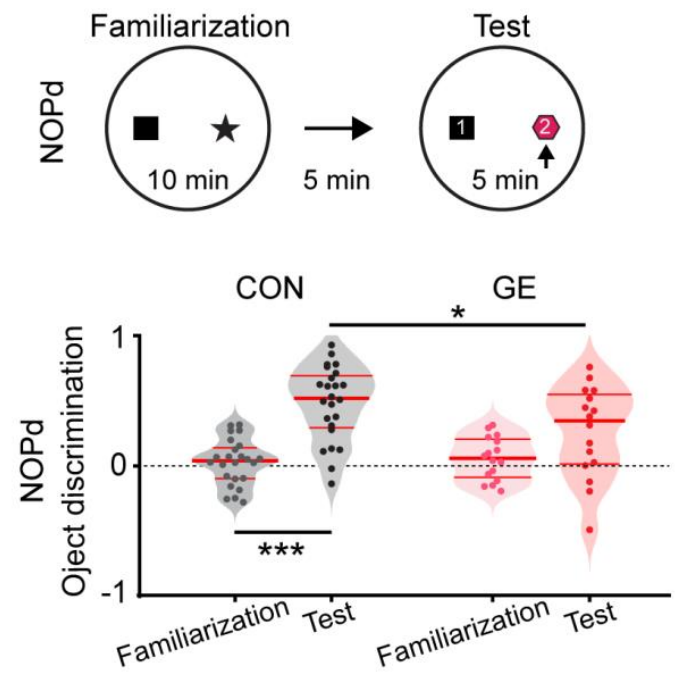

b
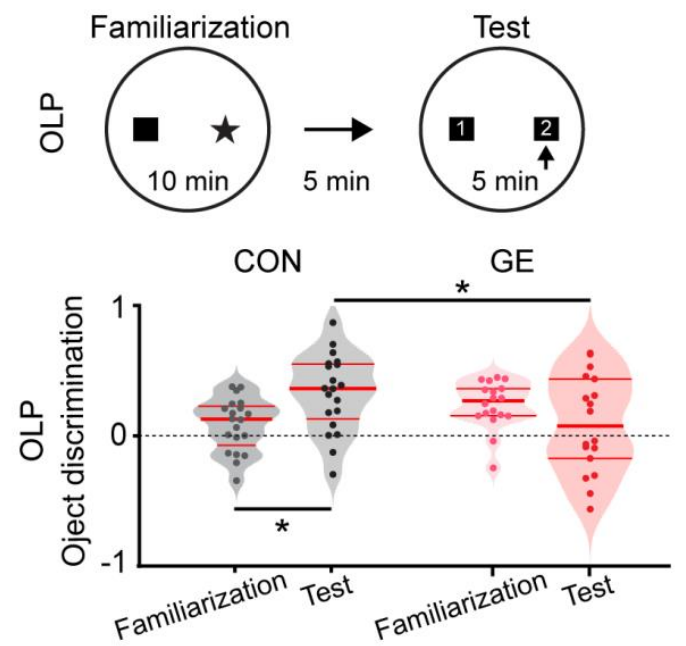

849 Fig. 1 The performance of pre-juvenile GE mice in associative recognition memory

850 tasks. (a) Schematic of the protocol for NOPd task (top) and violin plots displaying the

851 discrimination ratio in familiarization and test trials when averaged for CON and GE mice

852 (bottom). (b) Schematic of the protocol for OLP task (top) and violin plots displaying the 853 discrimination ratio in familiarization and test trials when averaged for CON and GE mice 854 (bottom). In (a) and (b), the black dotted line indicates chance level. Single data points are 855 shown as dots and the red horizontal lines in violin plots correspond to the median and the $85625^{\text {th }}$ and $75^{\text {th }}$ percentiles. ${ }^{*} \mathrm{p}<0.05,{ }^{* * *} \mathrm{p}<0.0001$. 
a
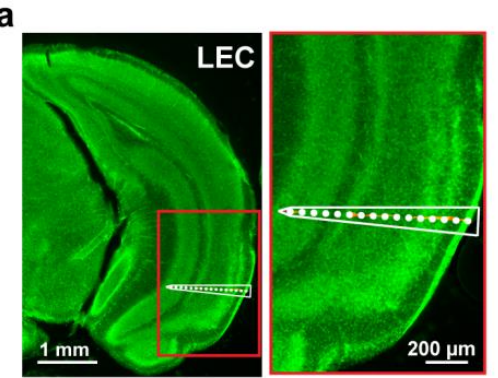

b
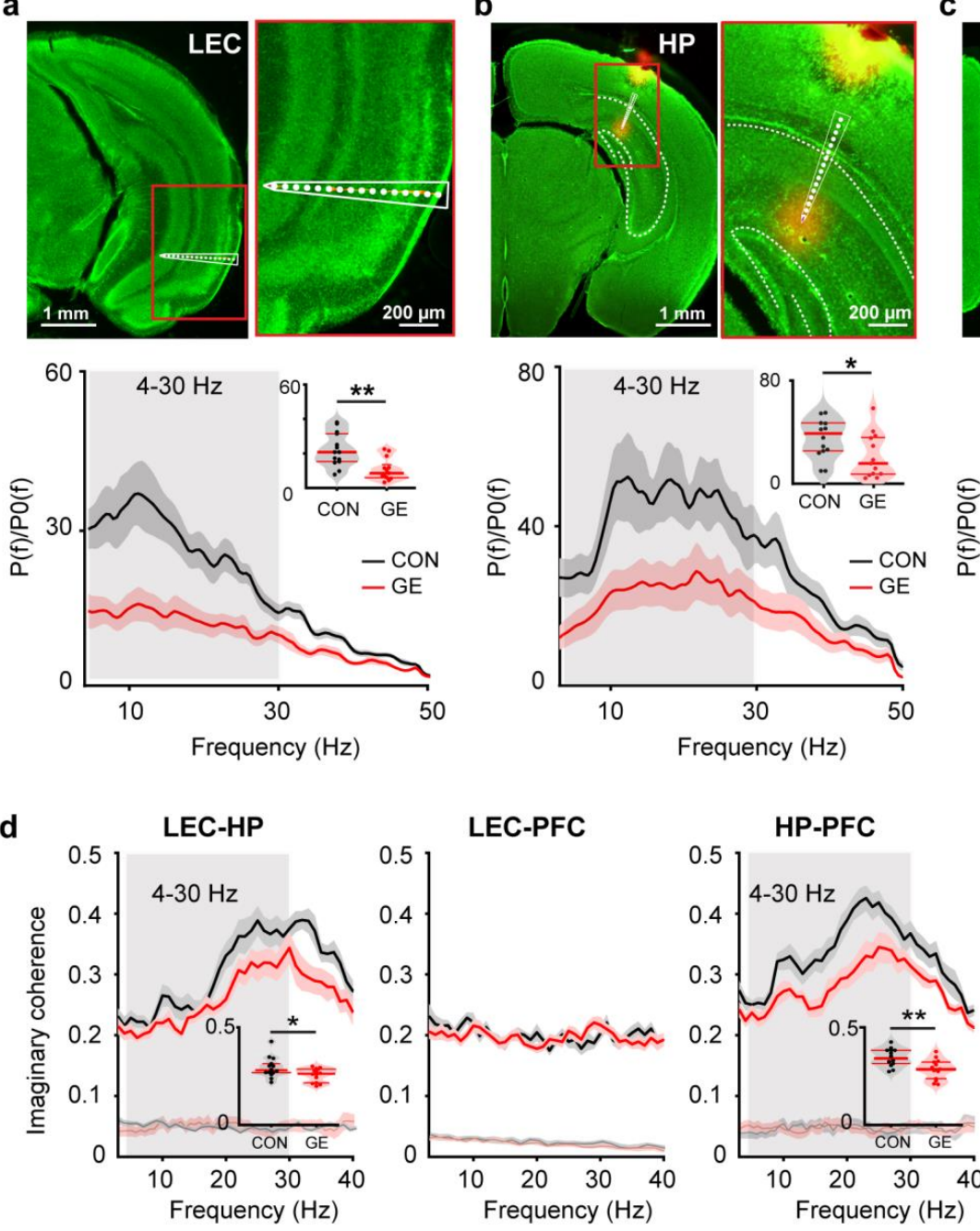

e
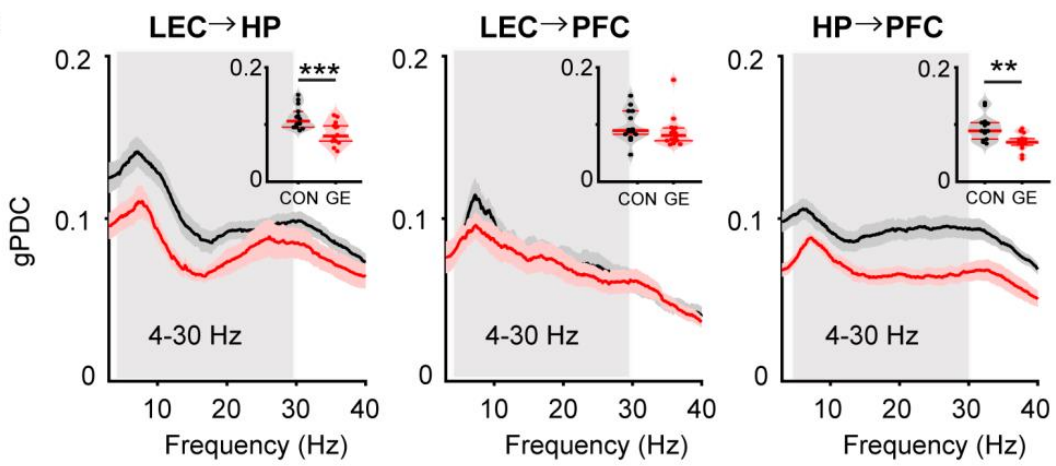
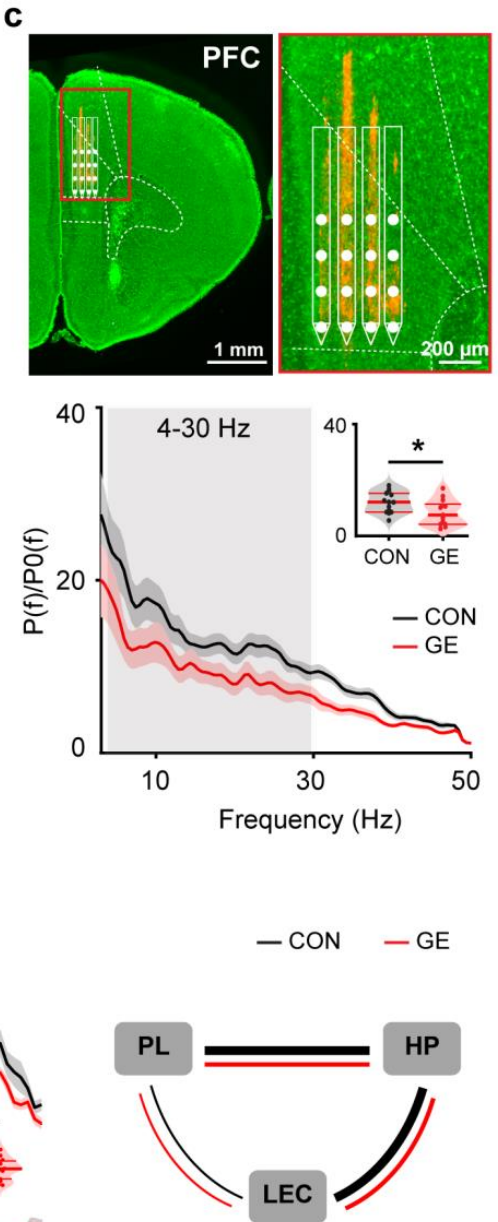

Fig. 2 Patterns of network activity in LEC, HP and PFC as well as functional communication within LEC-HP-PFC networks from neonatal CON and GE mice. (a) Digital photomontage reconstructing the location of the Dil-labeled $1 \times 16$-site recording 861 electrode (orange) in a $100 \mu$ m-thick coronal section containing the LEC from a P9 mouse. 862 Right, the position of recording sites (white dots) over LEC layers when displayed at higher 863 magnification. Bottom, averaged power spectra $P(f)$ of discontinuous oscillatory activity normalized to the baseline power $\mathrm{PO}(\mathfrak{f})$ of time windows lacking oscillatory activity in CON 
865 and GE mice. Inset, violin plots displaying the average power spectra from 1-50 Hz in CON 866 and GE mice. (b) Same as (a) for HP. (c) Same as (a) for PFC. (d) Line plots of mean 867 imaginary coherence for oscillatory activity simultaneously recorded in the LEC and HP, LEC 868 and PFC, as well as HP and PFC of CON (black) and GE (red) mice. The bottom lines in the 869 coherence plots correspond to the imaginary coherence calculated from shuffled data. Insets 870 for each coherence plot, violin plots displaying the imaginary coherence when averaged for $8714-30 \mathrm{~Hz}$. Right, schematic of synchrony within LEC-HP-PFC networks during neonatal 872 development as resolved by imaginary coherence. The line thickness indicates the strength 873 of the coupling between brain regions. (e) Same as (d) when the directional coupling within 874 LEC-HP-PFC networks was estimated by gPDC. Single data points are shown as dots and 875 the red horizontal bars in violin plots correspond to the median and the $25^{\text {th }}$ and $75^{\text {th }}$ 876 percentiles. ${ }^{*} p<0.05,{ }^{* *} p<0.01,{ }^{* * *} p<0.001$. 
a

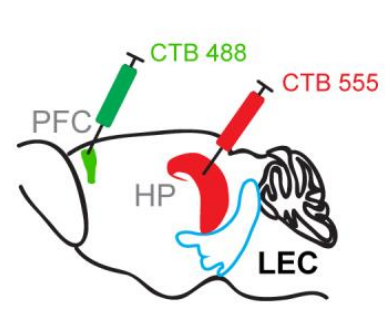

b

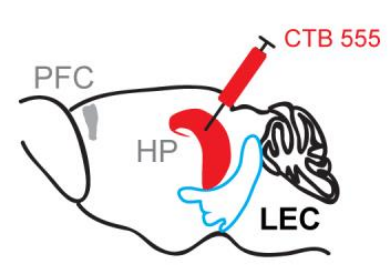

C

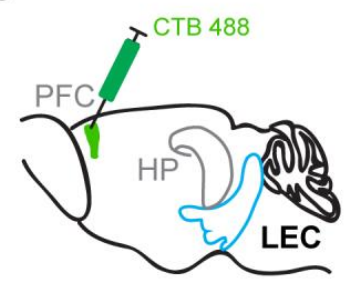

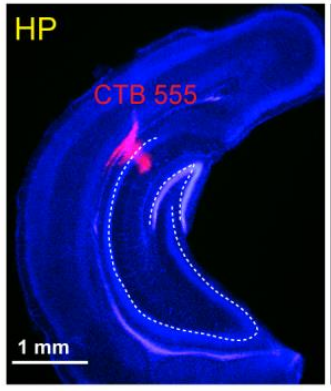
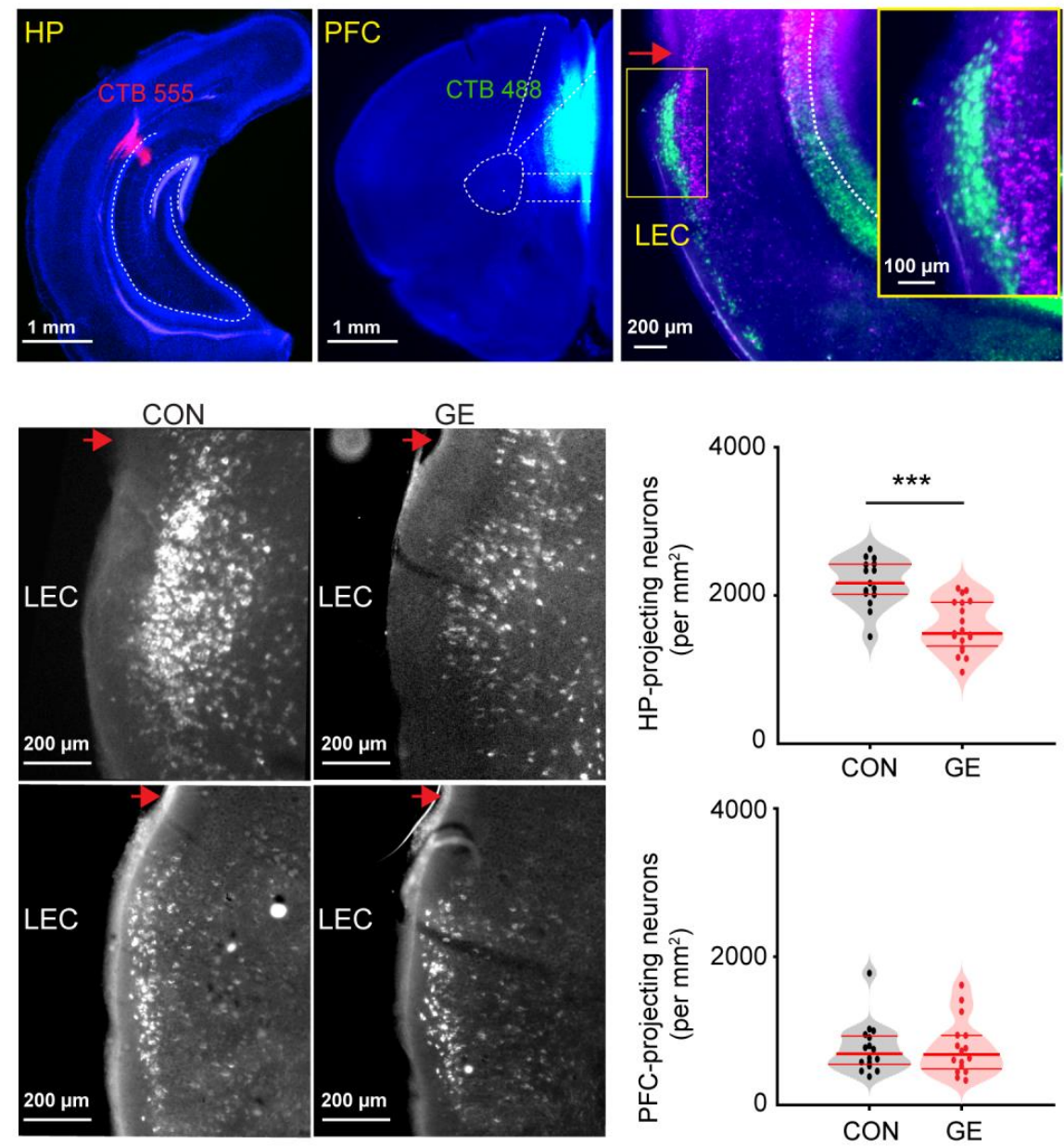

Fig.3 Long-range monosynaptic axonal projections connecting neonatal LEC, hippocampal CA1, and PFC. (a) Schematic of the retrograde tracer CTB488 injection in PFC and CTB555 injection in HP. Digital photomontage showing the CTB555 injection in the HP (left) and CTB488 injection in the PFC (middle) of a P10 CON mouse. Right, digital photomontage displaying CTB555- (red) and CTB488- (green) labeled neurons in LEC of the same mouse. Inset, labeled neurons in LEC shown at higher-magnification. The red arrow indicates rhinal fissure. (b) Left, schematic of the retrograde tracer CTB555 injection in HP. Middle, photographs depicting CTB555-labeled neurons (white dot) in the LEC of a P10 CON and GE mouse. Right, violin plot displaying the number of CTB555-labeled neurons in the LEC of CON and GE mice. (c) Same as (b) for CTB488 injection in PFC. Single data points (i.e. density of labeled neurons in LEC / slice) are shown as dots and red horizontal bars in violin plots correspond to the median and the $25^{\text {th }}$ and $75^{\text {th }}$ percentiles. ${ }^{* *} p<0.001$. 
a
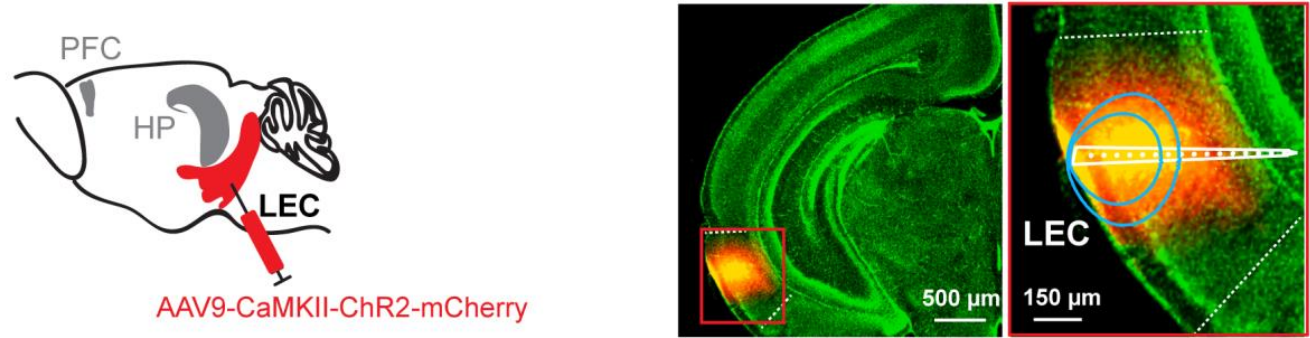

LEC

b 11111111111111111111111

HP

PFC
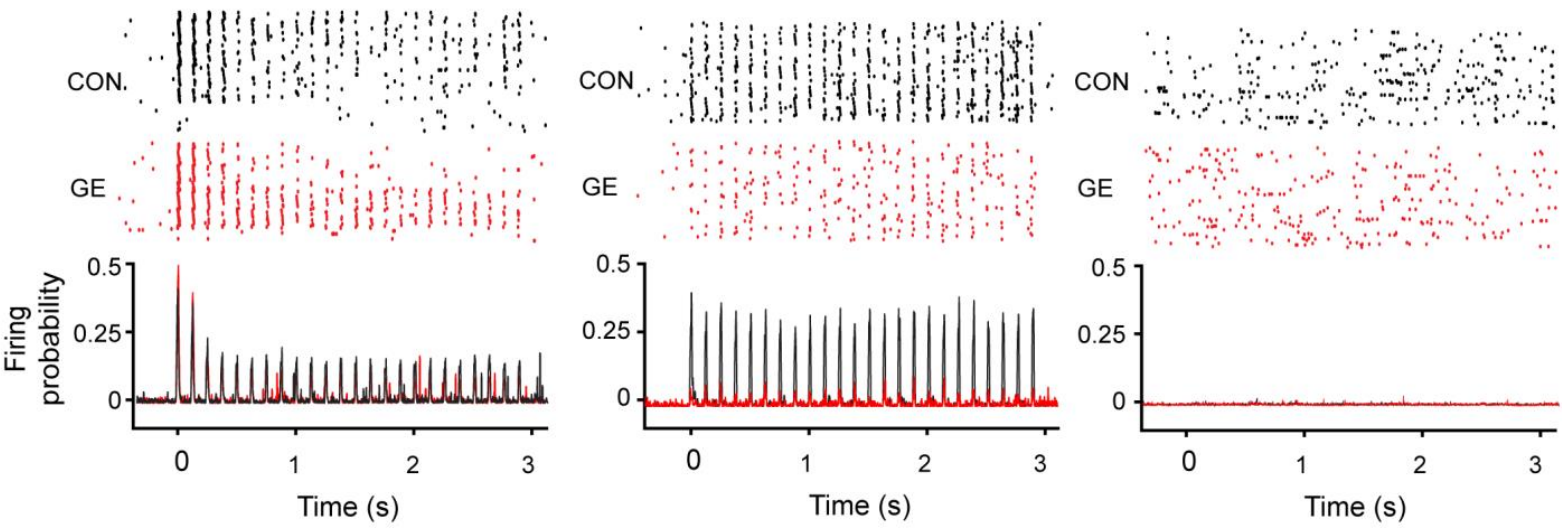

GE

GE
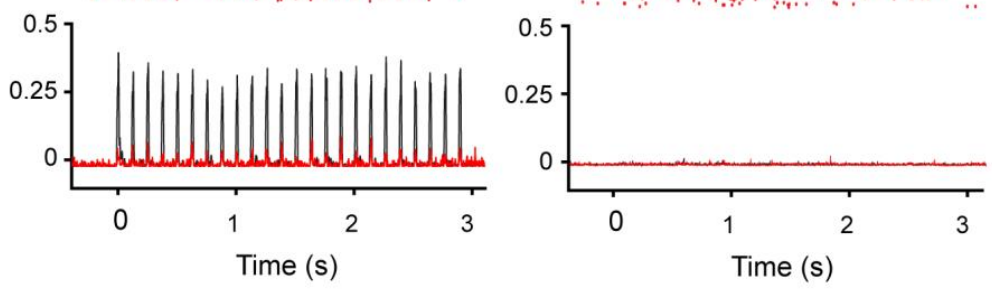

C

LEC-HP

LEC-PFC
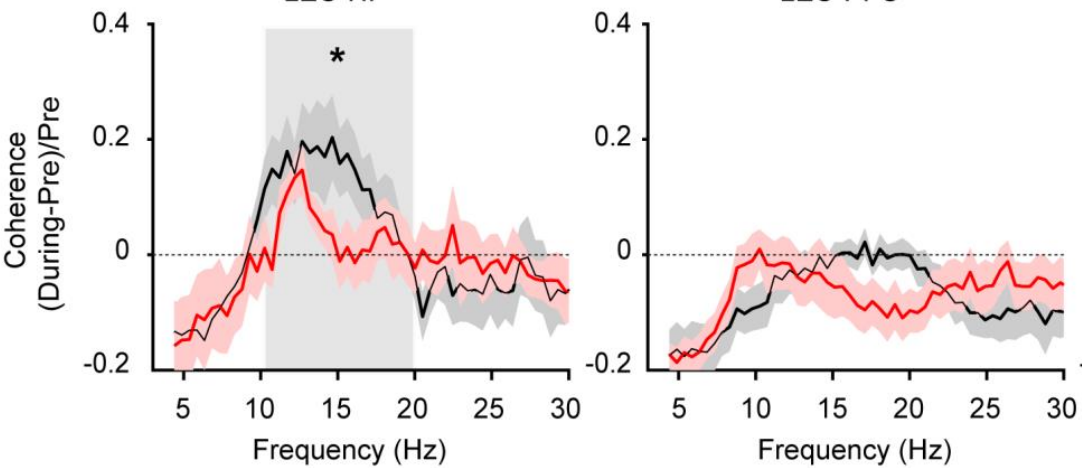

HP-PFC

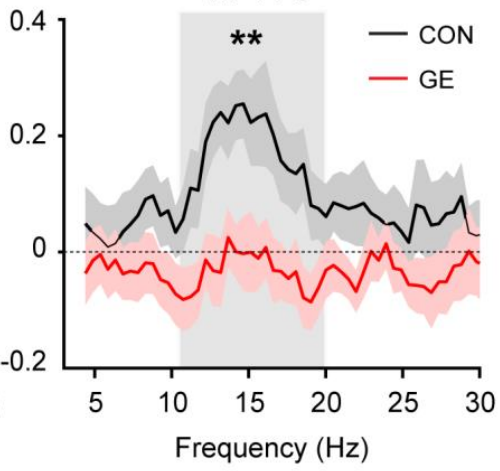

891

892

893

894

895

896

897

898

899

900

Fig. 4 Light-induced activation of LEC. (a) Schematic of AAV9-CaMKII-ChR2-mCherry injection in the LEC. Right, photographs depicting the injection position in the LEC of a P10 CON mouse and the position of injection site (red) shown at higher-magnification. Blue lines correspond to the iso-contour lines for light power of 1 and $10 \mathrm{~mW} / \mathrm{mm}^{2}$, respectively. (b) Representative raster plot and corresponding spike probability histogram for LEC, HP and PFC in response to 120 sweeps of $8 \mathrm{~Hz}$ pulse stimulation ( $3 \mathrm{~ms}$ pulse length, $473 \mathrm{~nm}$ ) in LEC. (c) Line plots of coherence between LEC and HP, LEC and PFC, and HP and PFC during ramp stimulation of LEC pyramidal neurons normalized to coherence values before stimulation. Single data points are shown as dots. ${ }^{*} \mathrm{p}<0.05,{ }^{* *} \mathrm{p}<0.01$. 
a
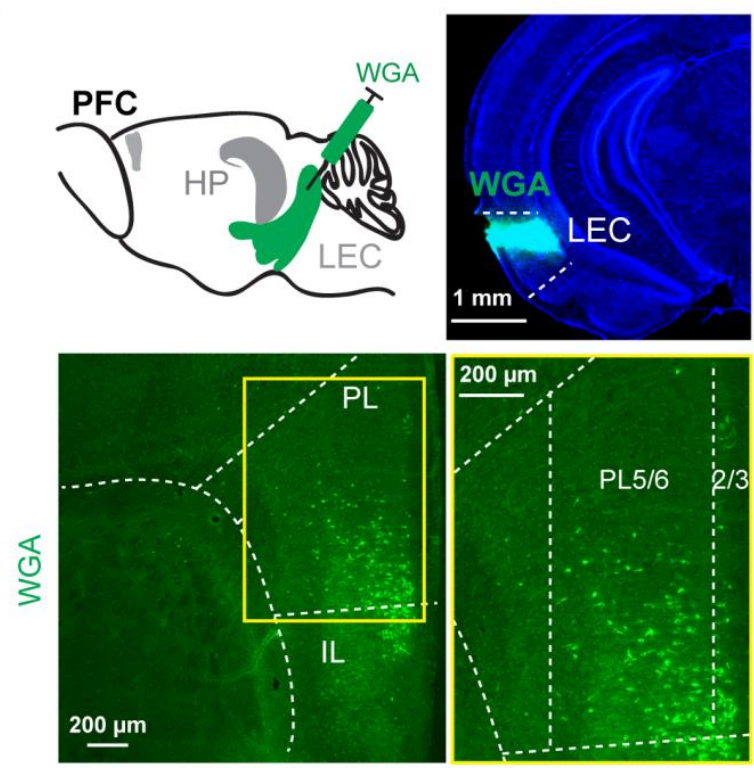

b
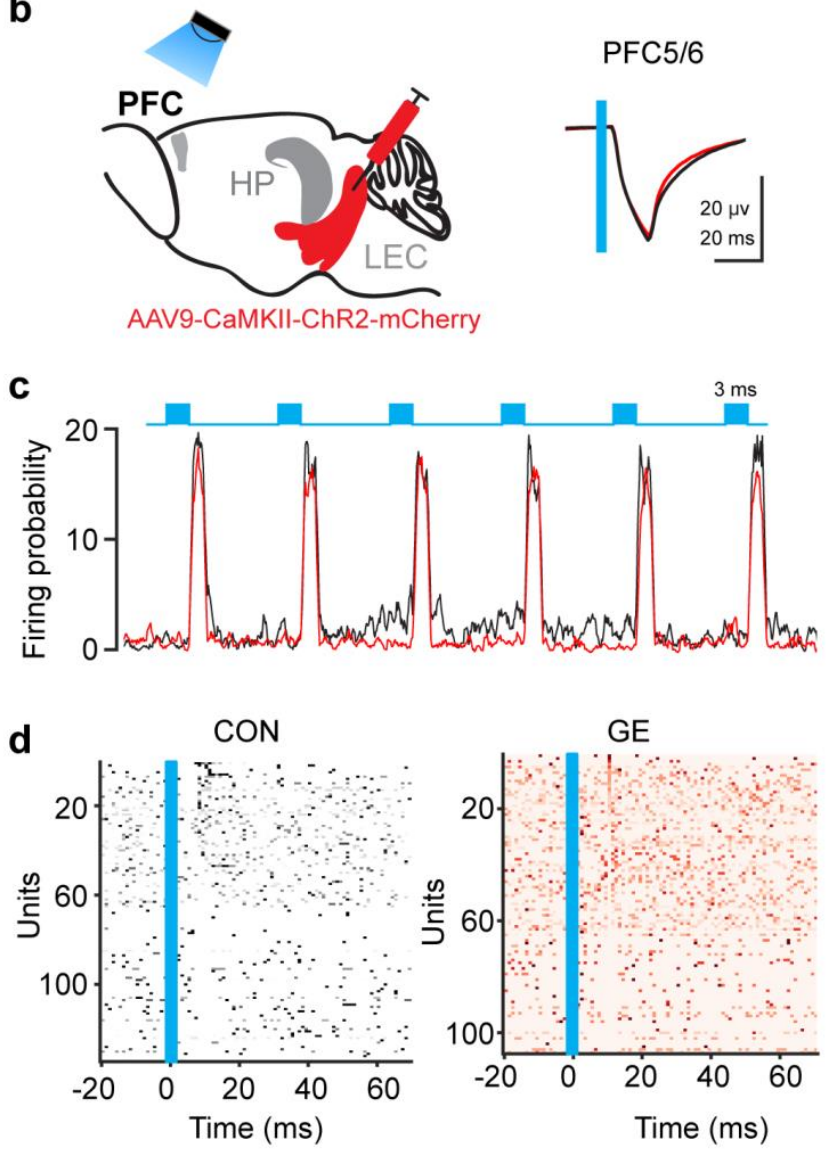

Fig. 5 Prelimbic neurons innervated by LEC and light-induced activation of LEC terminals in PFC. (a) Schematic of the anterograde trans-synaptic WGA injection in LEC. Photograph depicting the injection position in LEC of a P10 CON mouse. Bottom left, photographs depicting WGA-expressing neurons (green dots) in the PFC of a P10 CON mouse. Bottom right, WGA-labeled neurons in prelimbic layers displayed at a higher-magnification. PL, prelimbic subdivision of the PFC; IL, infralimbic subdivision of the PFC. (b) Schematic of the AAV9-CaMKII-ChR2-mCherry injection in the LEC and light stimulation of entorhinal axonal terminals in PFC. Right, averaged LFP traces recorded in the prelimbic layer $5 / 6$ in response to light stimulation of LEC terminals in CON (black) and GE (red) mice. The blue line indicates the $3 \mathrm{~ms}$-long pulse stimulation in PFC. (c) Spike probability histogram of prelimbic layer $5 / 6$ neurons in response to 120 sweeps of $8 \mathrm{~Hz}$ pulse stimulation ( $3 \mathrm{~ms}$ pulse length, $473 \mathrm{~nm}$, blue line) in CON (black) and GE mice (red). (d) Raster plot depicting the firing of single cells in response to the first pulse stimulation from each sweep in CON and GE groups. The blue line corresponds to the 3 ms-long pulse stimulation in PFC. 
a

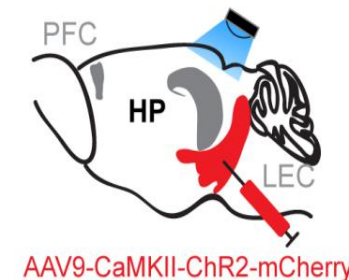

b
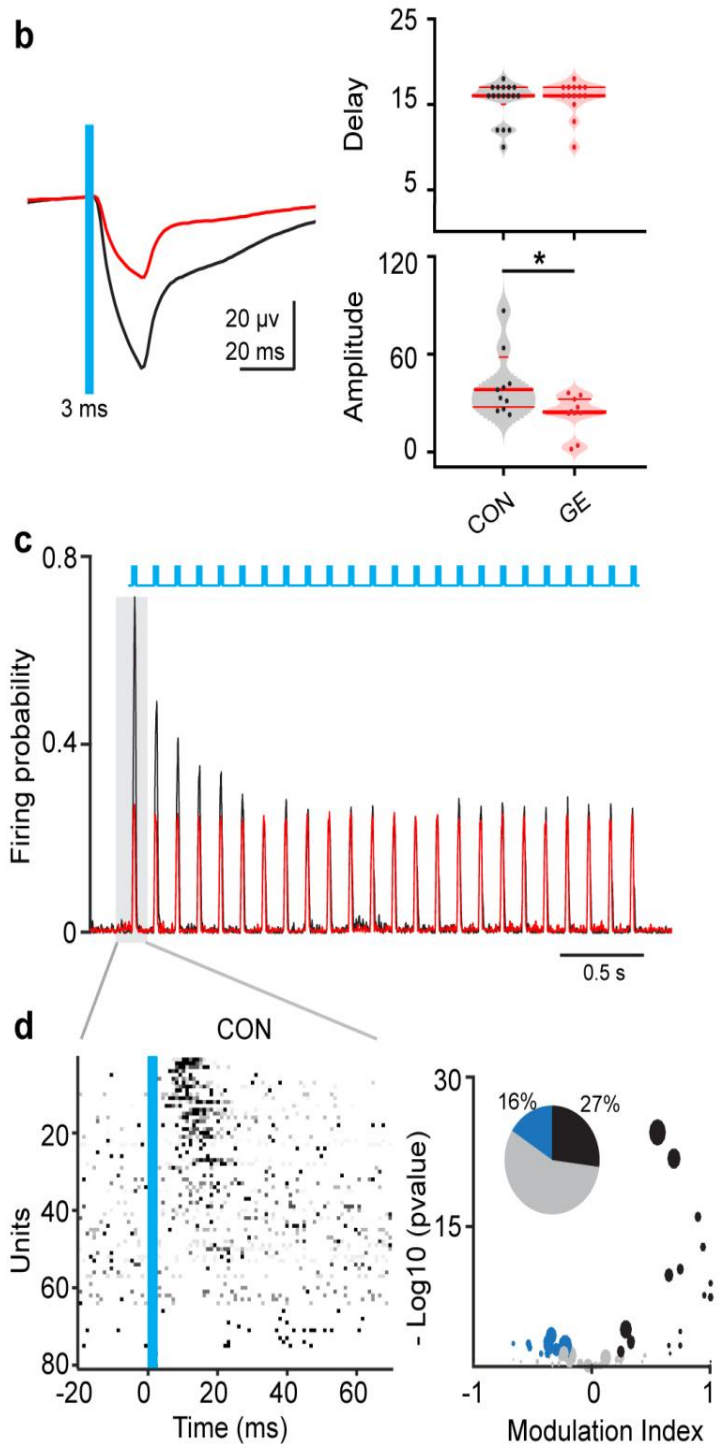

e

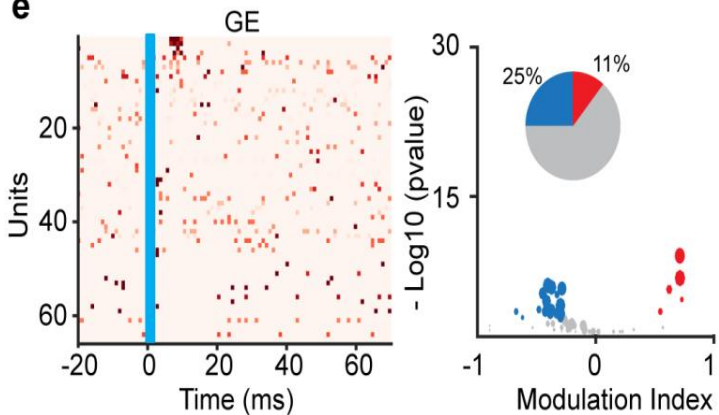

Fig. 6 Light-induced activation of LEC terminals in HP. (a) Schematic of the AAV9CaMKII-ChR2-mCherry injection in LEC and light stimulation of entorhinal axonal terminals in HP. Right, photographs depicting the LEC axonal terminals labeled by mCherry (red) in the HP of a P9 CON mouse. Blue lines correspond to the iso-contour lines for light power of 1 and $10 \mathrm{~mW} / \mathrm{mm}^{2}$, respectively. (b) Averaged LFP traces recorded in the HP in response to light stimulation of LEC terminals in CON (black) and GE (red) mice. The blue line indicates the 3 mslong pulse stimulation in HP. Right up, violin plots displaying the delay of the biggest response of the averaged LFP in HP. Right bottom, violin plots displaying the amplitude of the biggest response of the averaged LFP in HP. (c) Spike probability histogram for hippocampal neurons in response to 120 sweeps of $8 \mathrm{~Hz}$ pulse stimulation ( $3 \mathrm{~ms}$ pulse length, $473 \mathrm{~nm}$, blue line) in CON (black) and GE (red) mice. (d) Left, raster plot depicting the firing of single hippocampal cells in response to the first pulse stimulation from each sweep in CON group. Right, the modulation index of spiking response of hippocampal single units to pulse stimulation. Modulation index $>0$ indicates increased firing activity, whereas values $<0$ correspond to decreased firing activity. Inset, pie plot depicting the percentage of hippocampal single units activated (black), inhibited (blue), nonresponded (gray) to the stimulation. (e) Same as (d) for GE mice. Single data points are shown as dots and the red horizontal bars in violin plots correspond to the median and the $25^{\text {th }}$ and $75^{\text {th }}$ percentiles. ${ }^{*} \mathrm{p}<0.05$. 
a

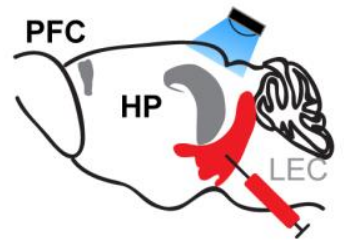

AAV9-CaMKII-ChR2-mCherry

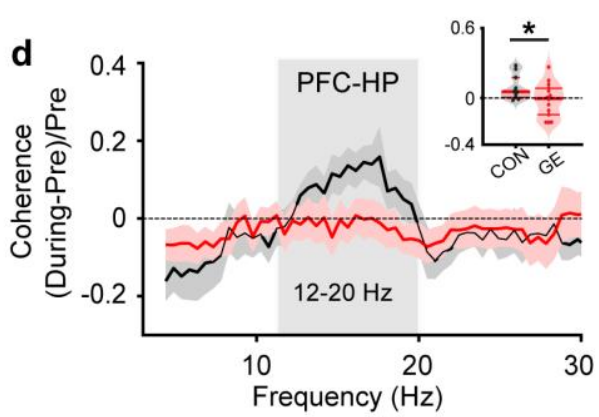

b
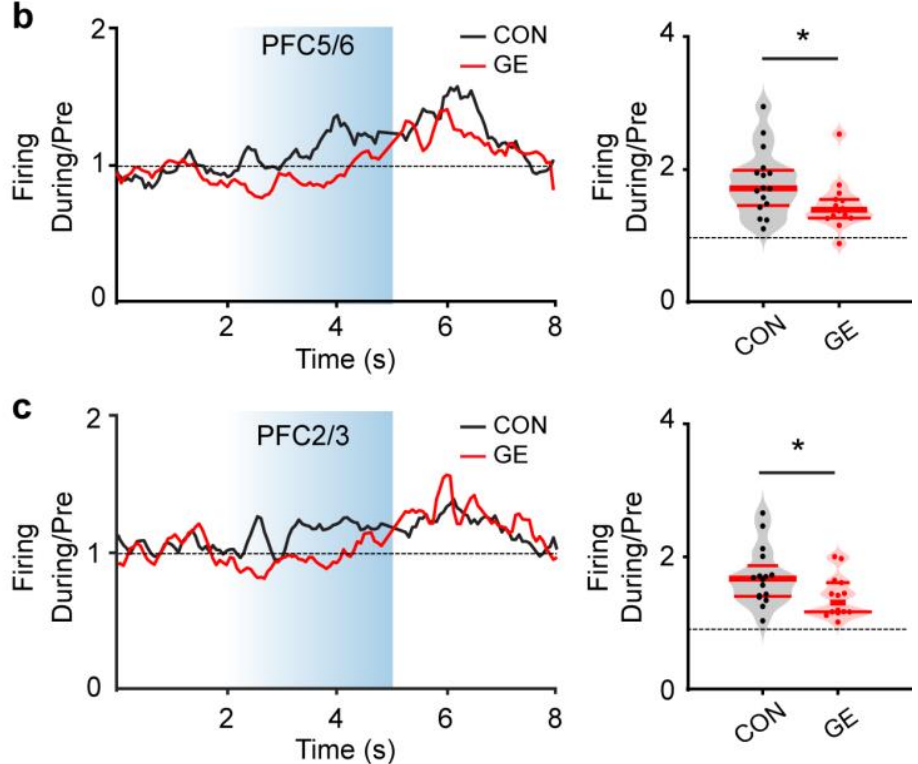

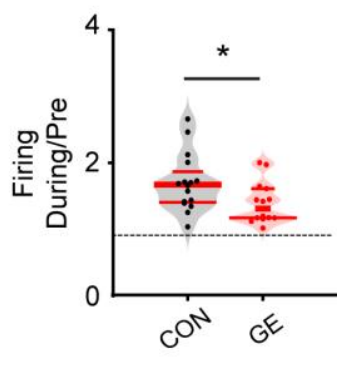

970

971

972

973

974

975

976

977

978

979

980

981

982

983

Fig. 7 Firing activity in PFC during light-induced activation of LEC terminals in HP. (a) Schematic of AAV9-CaMKII-ChR2-mCherry injection in LEC and light stimulation of entorhinal axonal terminals in HP. (b) Line plot of firing activity of prelimbic layer $5 / 6$ neurons during 3 s-long ramp stimulation (light blue shadow) of LEC terminals in HP normalized to the activity before stimulation for CON and GE mice. The horizontal dotted line corresponds to no changes of firing activity during the stimulation. Right, violin plots displaying the firing activity of prelimbic layer5/6 neurons during $3 \mathrm{~s}$-long ramp stimulation normalized to the activity before stimulation. (c) Same as (b) for prelimbic layer 2/3 neurons. (d) Line plots of HP-PFC coherence calculated during ramp stimulation of entorhinal terminals in HP and normalized to coherence values before stimulation. Inset, violin plot displaying the averaged 12-20 Hz coherence between HP and PFC during stimulation when normalized to coherence values before stimulation. Single data points are shown as dots and the red horizontal bars in violin plots correspond to the median and the 25 th and 75 th percentiles. ${ }^{*} p<0.05,{ }^{* *} p<0.01$. 


\begin{tabular}{|c|c|c|c|c|c|c|c|}
\hline & & \multicolumn{3}{|c|}{ HP-projecting neurons } & \multicolumn{3}{|c|}{ PFC-projecting neurons } \\
\hline & & CON & GE & $p$ & CON & GE & $p$ \\
\hline \multirow{5}{*}{$\begin{array}{l}\text { Passive } \\
\text { properties }\end{array}$} & $\mathrm{RMP}(\mathrm{mV})$ & $-68.76 \pm 8.19$ & $-69.24 \pm 7.44$ & 0.12 & $-69.19 \pm 4.47$ & $-68.39 \pm 5.47$ & 0.99 \\
\hline & $\mathrm{Cm}(\mathrm{pF})$ & $111.85 \pm 21.99$ & $118.96 \pm 14.10$ & 0.46 & $120.32 \pm 22.02$ & $119.53 \pm 14.05$ & 0.96 \\
\hline & $\operatorname{Rin}(\mathrm{M} \Omega)$ & $606.99 \pm 181.96$ & $632.35 \pm 102.39$ & 0.62 & $481.20 \pm 96.20$ & $551.86 \pm 93.68$ & 0.27 \\
\hline & Tm (ms) & $67.24 \pm 14.21$ & $72.86 \pm 16.08$ & 0.17 & $58.95 \pm 12.47$ & $68.01 \pm 12.95$ & 0.29 \\
\hline & AP threshold (mV) & $-41.16 \pm 7.06$ & $-42.20 \pm 2.84$ & 0.71 & $-42.94 \pm 2.61$ & $-44.05 \pm 2.57$ & 0.57 \\
\hline \multirow{4}{*}{$\begin{array}{l}\text { Active } \\
\text { Properties }\end{array}$} & AP amplitude (mV) & $79.33 \pm 5.29$ & $77.88 \pm 2.71$ & 0.49 & $80.80 \pm 3.38$ & $81.21 \pm 2.94$ & 0.85 \\
\hline & Half-width (ms) & $2.82 \pm 0.45$ & $2.61 \pm 0.26$ & 0.16 & $2.07 \pm 0.31$ & $1.92 \pm 0.45$ & 0.61 \\
\hline & Rheobase (pA) & $45.61 \pm 20.87$ & $54.20 \pm 16.42$ & 0.69 & $56.07 \pm 16.41$ & $50.53 \pm 8.27$ & 0.49 \\
\hline & Firing frequency $(\mathrm{Hz})$ & $14.39 \pm 4.53$ & $14.79 \pm 1.94$ & 0.82 & $13.81 \pm 3.05$ & $16.67 \pm 3.16$ & 0.18 \\
\hline
\end{tabular}


Figures

a
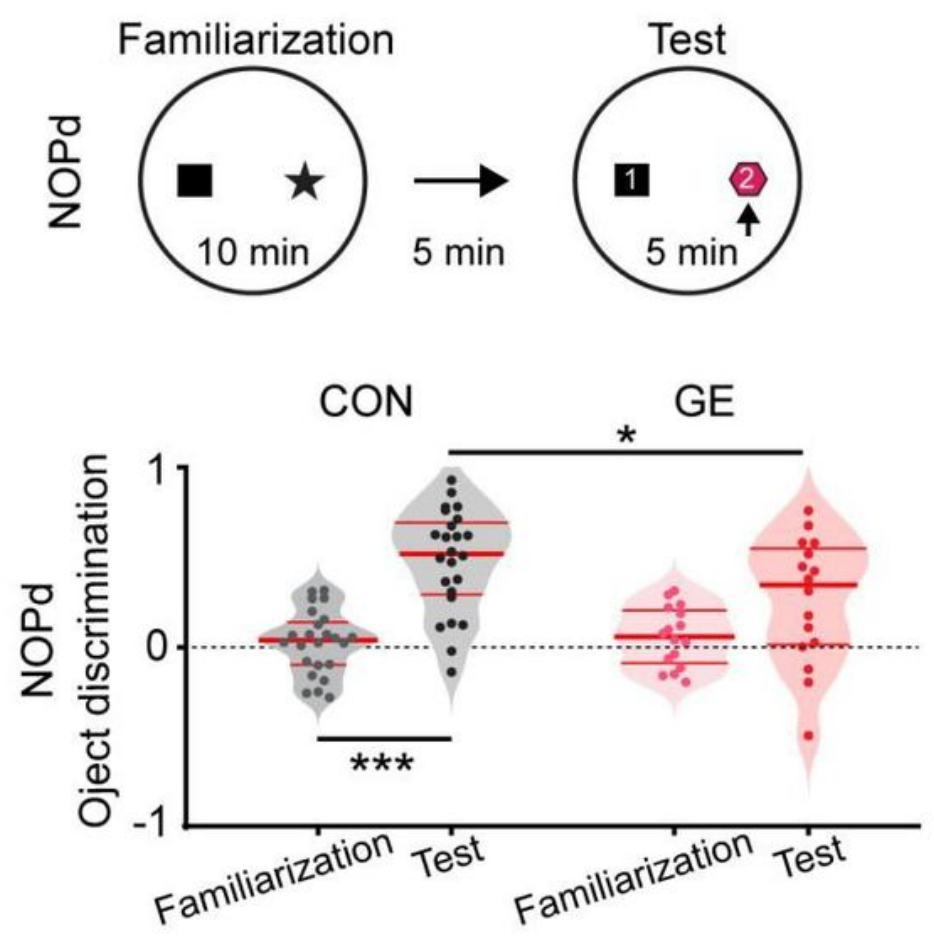

b
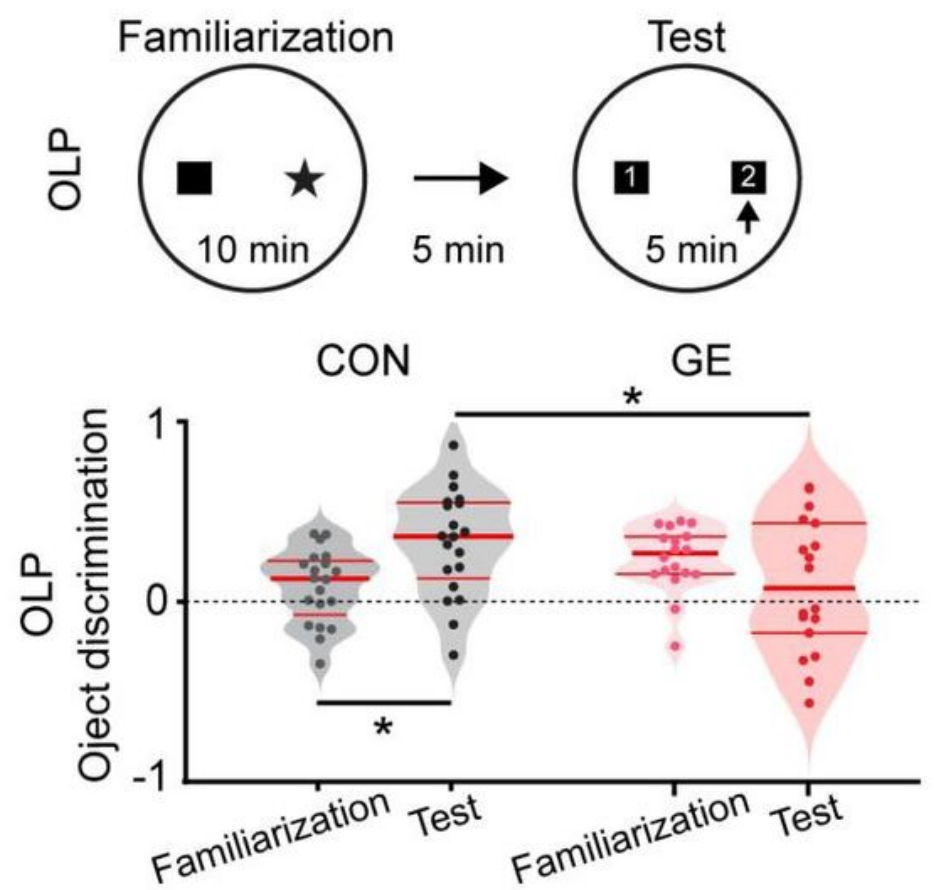

Figure 1

The performance of pre-juvenile GE mice in associative recognition memory tasks. (a) Schematic of the protocol for NOPd task (top) and violin plots displaying the discrimination ratio in familiarization and test trials when averaged for CON and GE mice (bottom). (b) Schematic of the protocol for OLP task (top) and 
violin plots displaying the discrimination ratio in familiarization and test trials when averaged for CON and GE mice (bottom). In (a) and (b), the black dotted line indicates chance level. Single data points are shown as dots and the red horizontal lines in violin plots correspond to the median and the 25th and 75th percentiles. ${ }^{*} p<0.05,{ }^{* *} p<0.0001$.

a
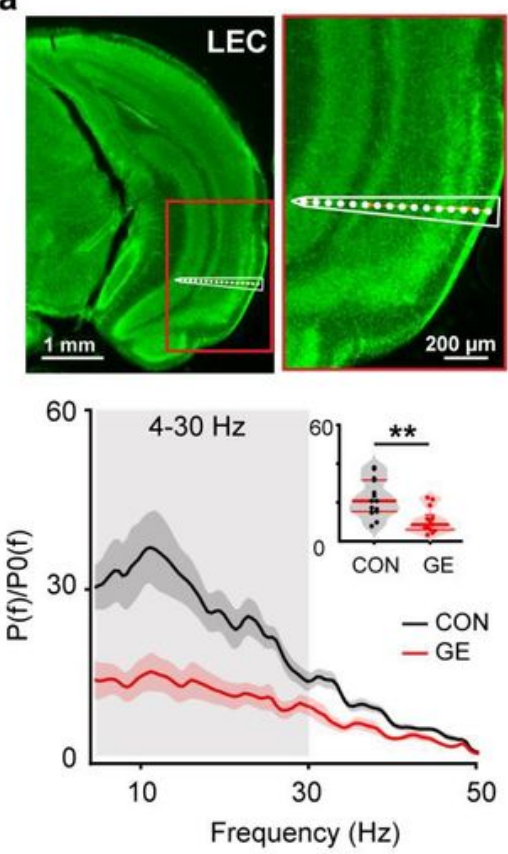

b
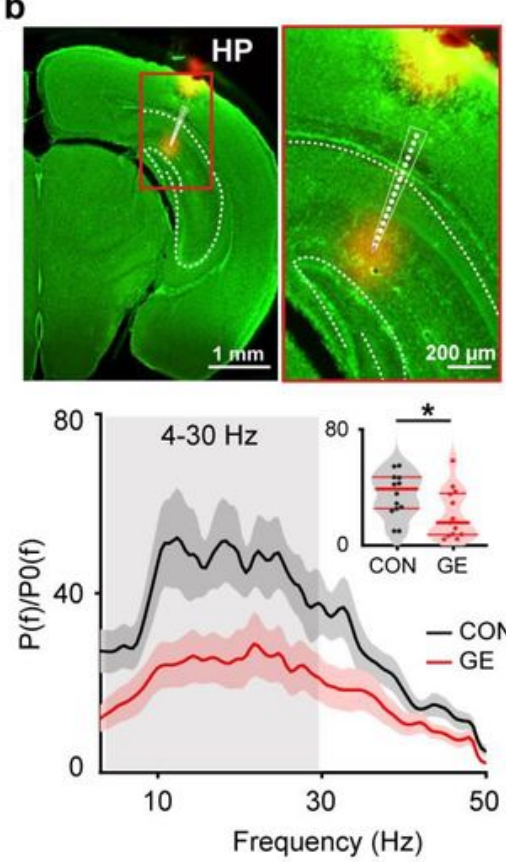

LEC-PFC
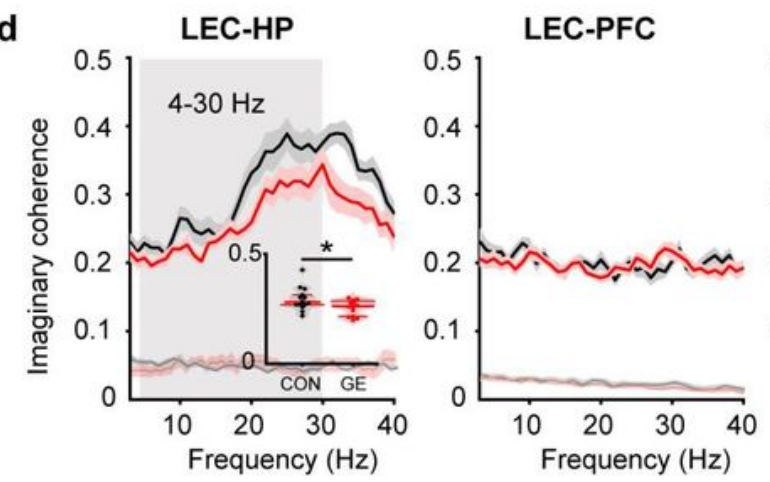

e

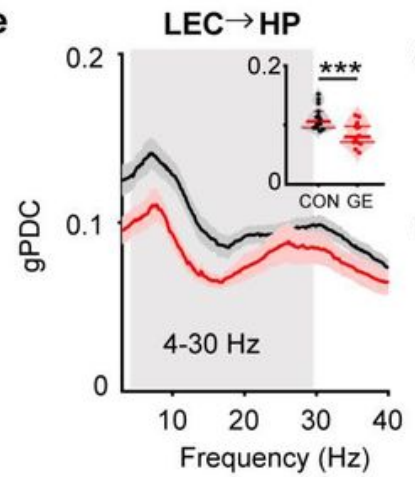

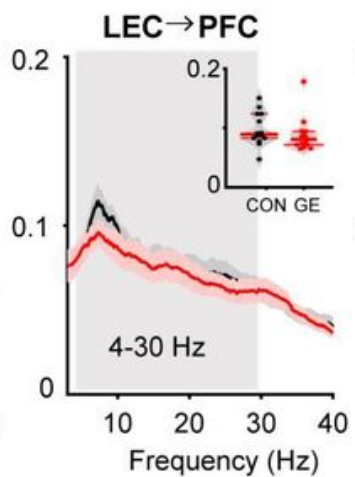
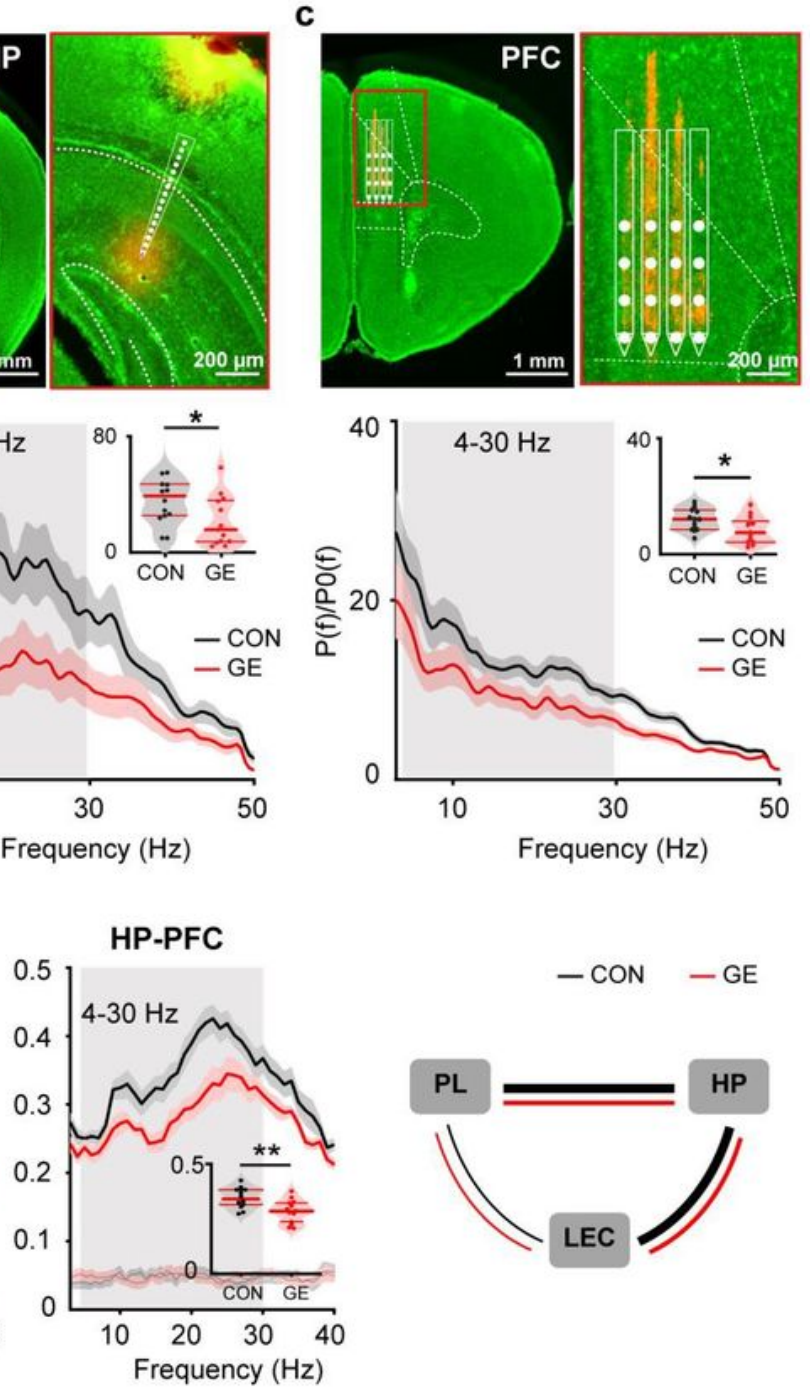

Frequency $(\mathrm{Hz})$
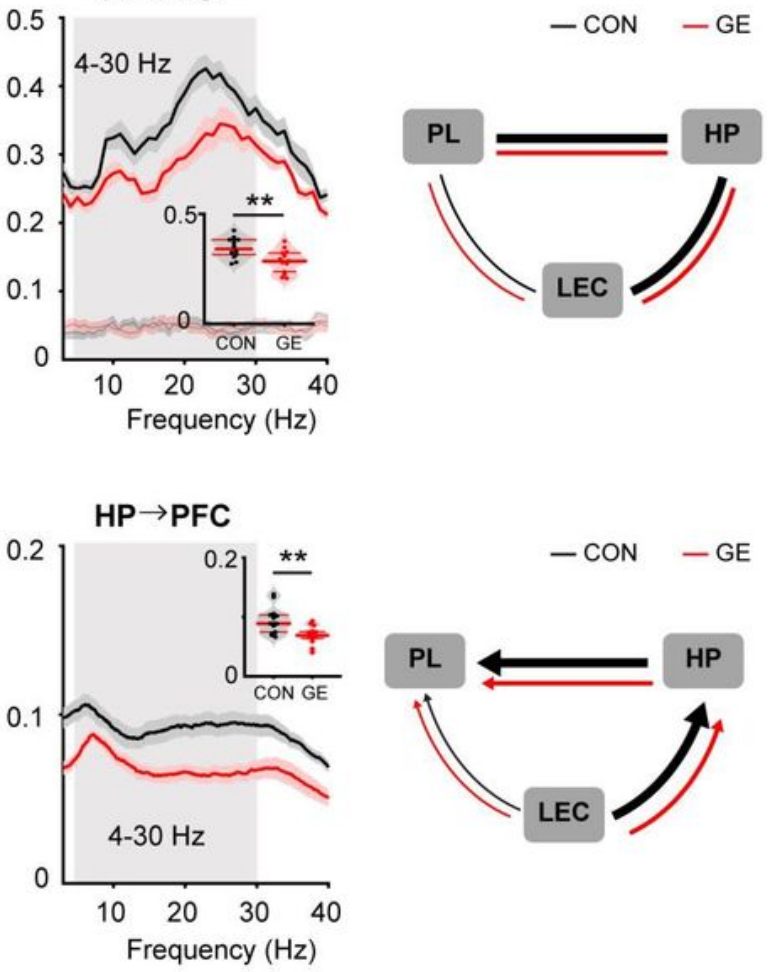

Figure 2 
Patterns of network activity in LEC, HP and PFC as well as functional communication within LEC-HP-PFC networks from neonatal CON and GE mice. (a) Digital photomontage reconstructing the location of the Dil-labeled $1 \times 16$-site recording electrode (orange) in a $100 \mu \mathrm{m}$-thick coronal section containing the LEC from a P9 mouse. Right, the position of recording sites (white dots) over LEC layers when displayed at higher magnification. Bottom, averaged power spectra $P(f)$ of discontinuous oscillatory activity normalized to the baseline power $\mathrm{PO}(\mathrm{f})$ of time windows lacking oscillatory activity in CON and GE mice. Inset, violin plots displaying the average power spectra from 1-50 Hz in CON and GE mice. (b) Same as (a) for HP. (c) Same as (a) for PFC. (d) Line plots of mean imaginary coherence for oscillatory activity simultaneously recorded in the LEC and HP, LEC and PFC, as well as HP and PFC of CON (black) and GE (red) mice. The bottom lines in the coherence plots correspond to the imaginary coherence calculated from shuffled data. Insets for each coherence plot, violin plots displaying the imaginary coherence when averaged for 4-30 Hz. Right, schematic of synchrony within LEC-HP-PFC networks during neonatal development as resolved by imaginary coherence. The line thickness indicates the strength of the coupling between brain regions. (e) Same as (d) when the directional coupling within LEC-HP-PFC networks was estimated by gPDC. Single data points are shown as dots and the red horizontal bars in violin plots correspond to the median and the 25th and 75th percentiles. ${ }^{*} p<0.05,{ }^{* \star} p<0.01,{ }^{\star \star *} p<0.001$. 
a

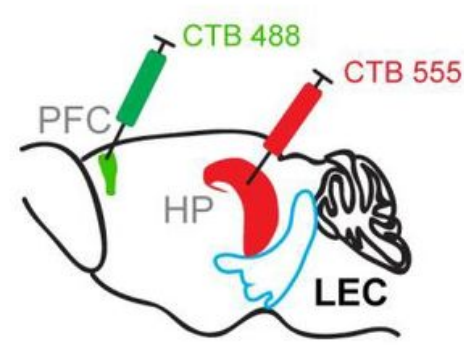

b

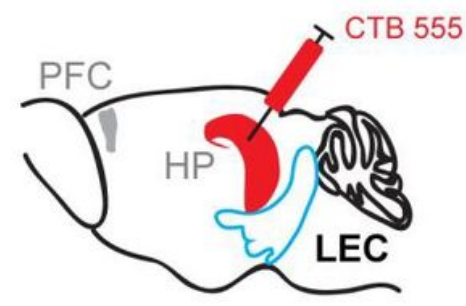

C

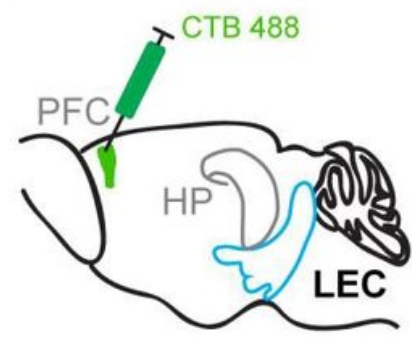

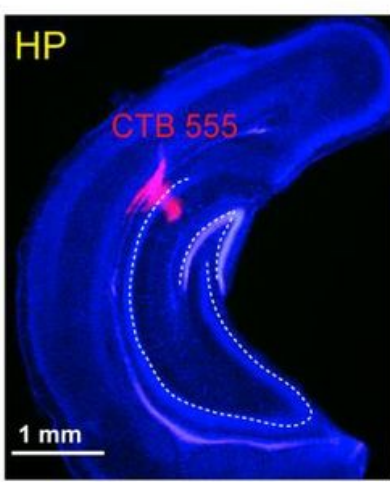
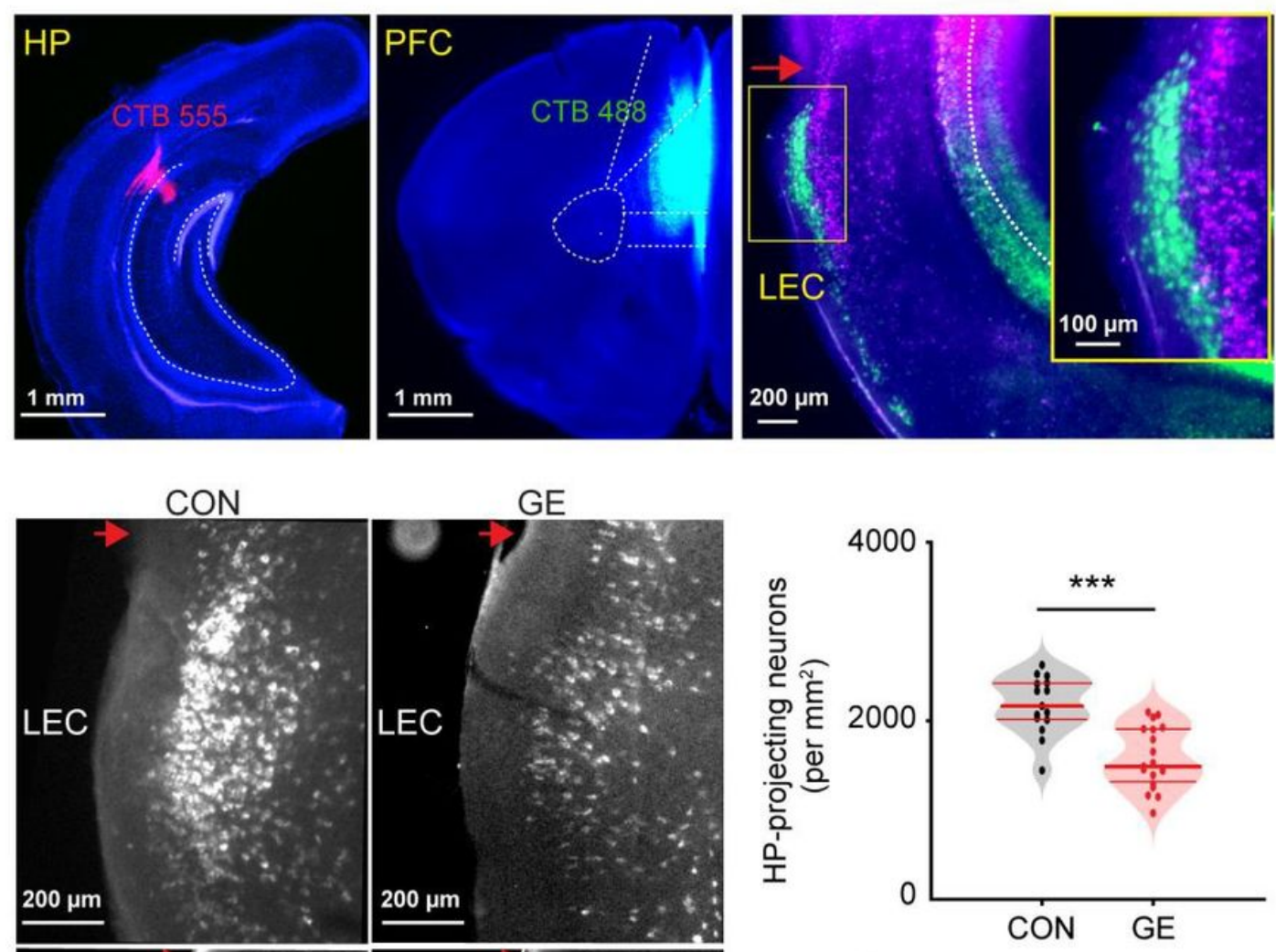

GE
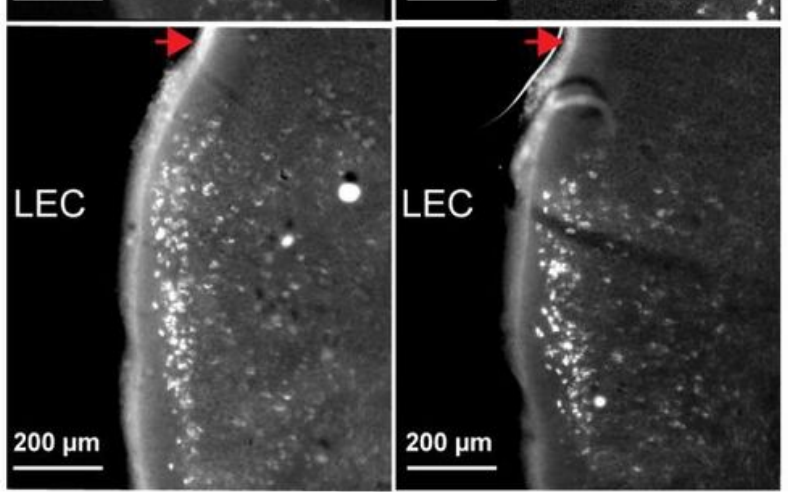
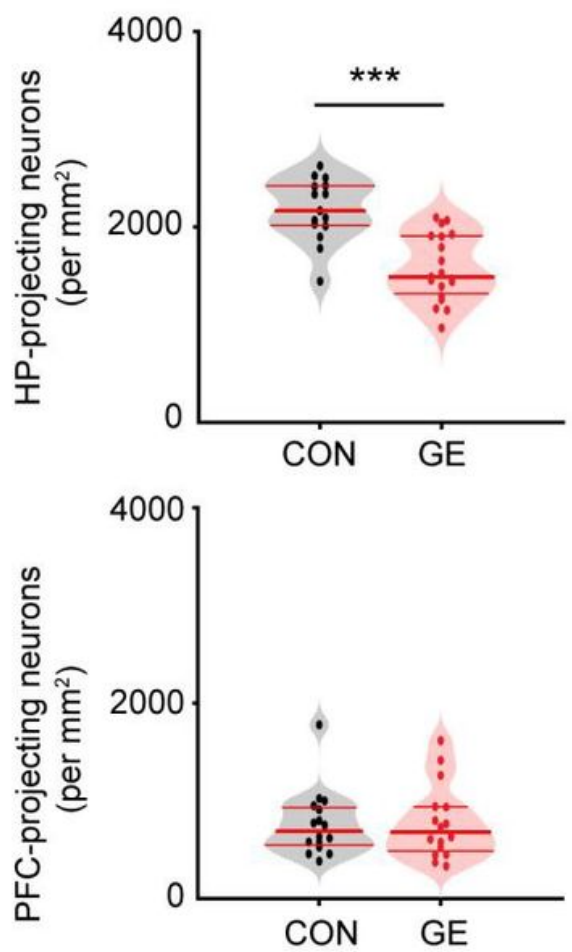

Figure 3

Long-range monosynaptic axonal projections connecting neonatal LEC, hippocampal CA1, and PFC. (a) Schematic of the retrograde tracer CTB488 injection in PFC and CTB555 injection in HP. Digital photomontage showing the CTB555 injection in the HP (left) and CTB488 injection in the PFC (middle) of a P10 CON mouse. Right, digital photomontage displaying CTB555- (red) and CTB488- (green) labeled neurons in LEC of the same mouse. Inset, labeled neurons in LEC shown at higher-magnification. The red arrow indicates rhinal fissure. (b) Left, schematic of the retrograde tracer CTB555 injection in HP. Middle, photographs depicting CTB555-labeled neurons (white dot) in the LEC of a P10 CON and GE mouse. Right, violin plot displaying the number of CTB555-labeled neurons in the LEC of CON and GE mice. (c) Same as (b) for CTB488 injection in PFC. Single data points (i.e. density of labeled neurons in LEC / slice) 
are shown as dots and red horizontal bars in violin plots correspond to the median and the 25th and 75th percentiles. ${ }^{* \star *} \mathrm{p}<0.001$.

a
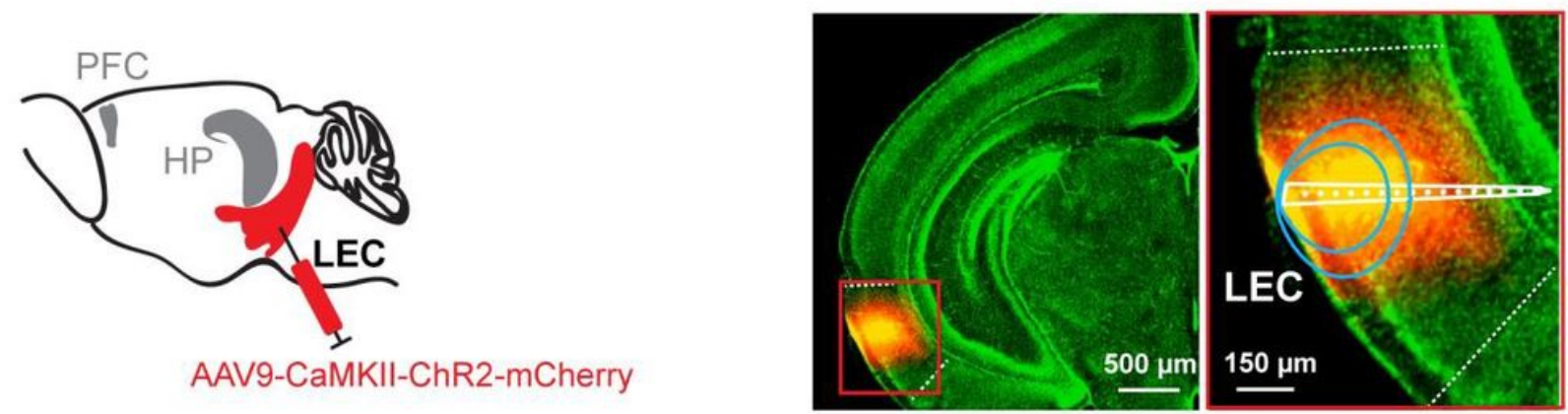

LEC

b
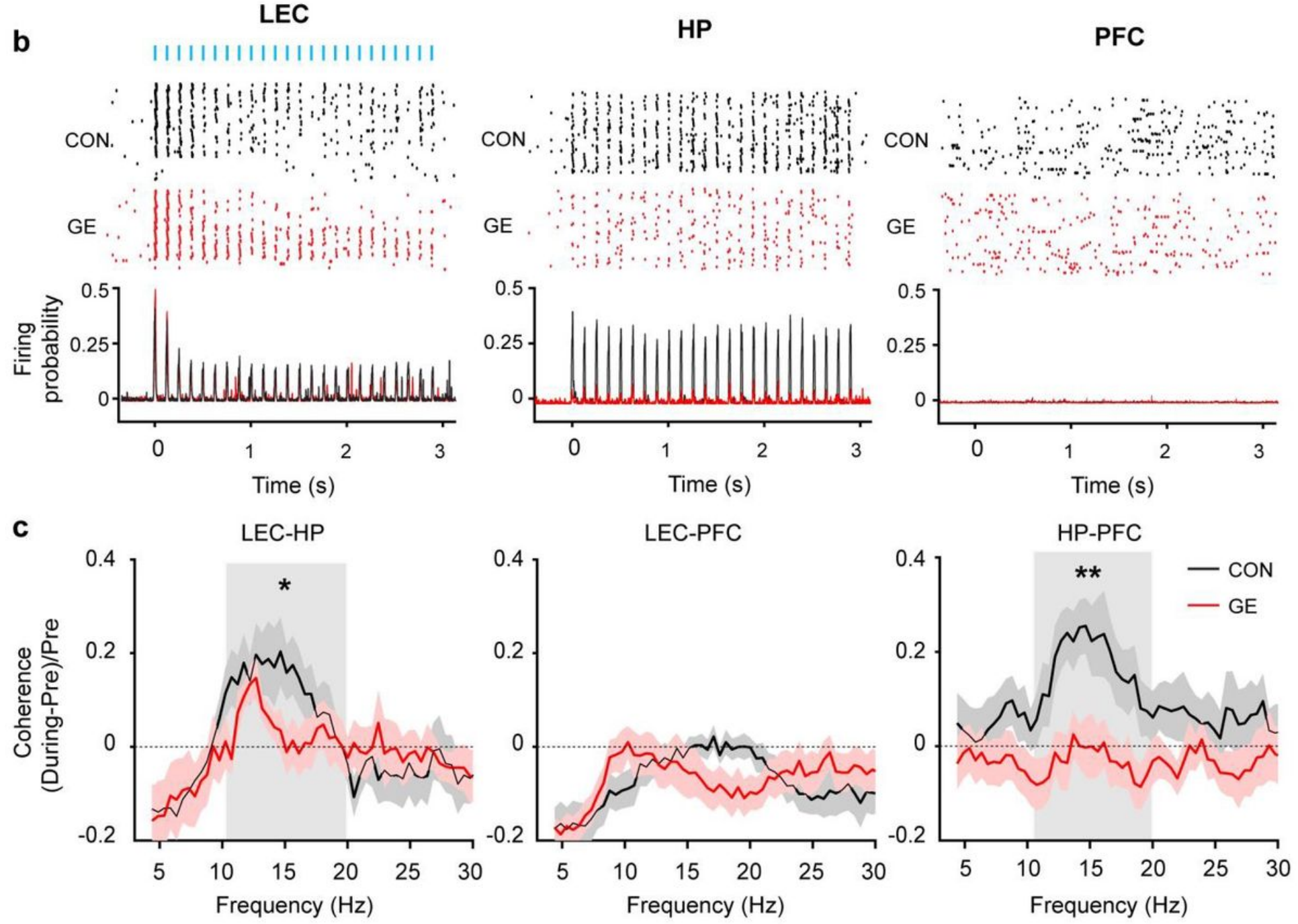

\section{Figure 4}

Light-induced activation of LEC. (a) Schematic of AAV9-CaMKII-ChR2-mCherry injection in the LEC. Right, photographs depicting the injection position in the LEC of a P10 CON mouse and the position of injection site (red) shown at higher-magnification. Blue lines correspond to the iso-contour lines for light power of 1 and $10 \mathrm{~mW} / \mathrm{mm} 2$, respectively. (b) Representative raster plot and corresponding spike probability 
histogram for LEC, HP and PFC in response to 120 sweeps of $8 \mathrm{~Hz}$ pulse stimulation (3 ms pulse length, $473 \mathrm{~nm}$ ) in LEC. (c) Line plots of coherence between LEC and HP, LEC and PFC, and HP and PFC during ramp stimulation of LEC pyramidal neurons normalized to coherence values before stimulation. Single data points are shown as dots. ${ }^{*} p<0.05,{ }^{*} \mathrm{p}<0.01$.

a
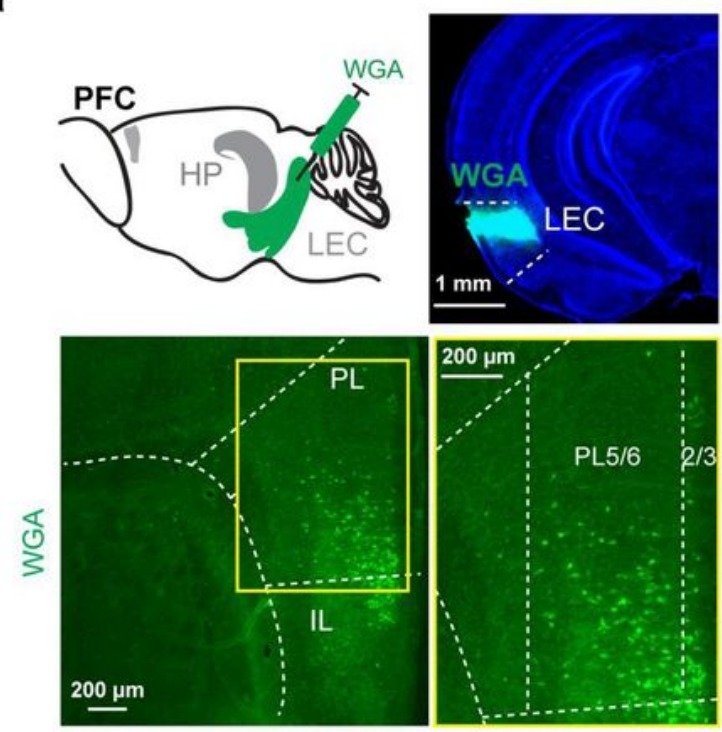

b
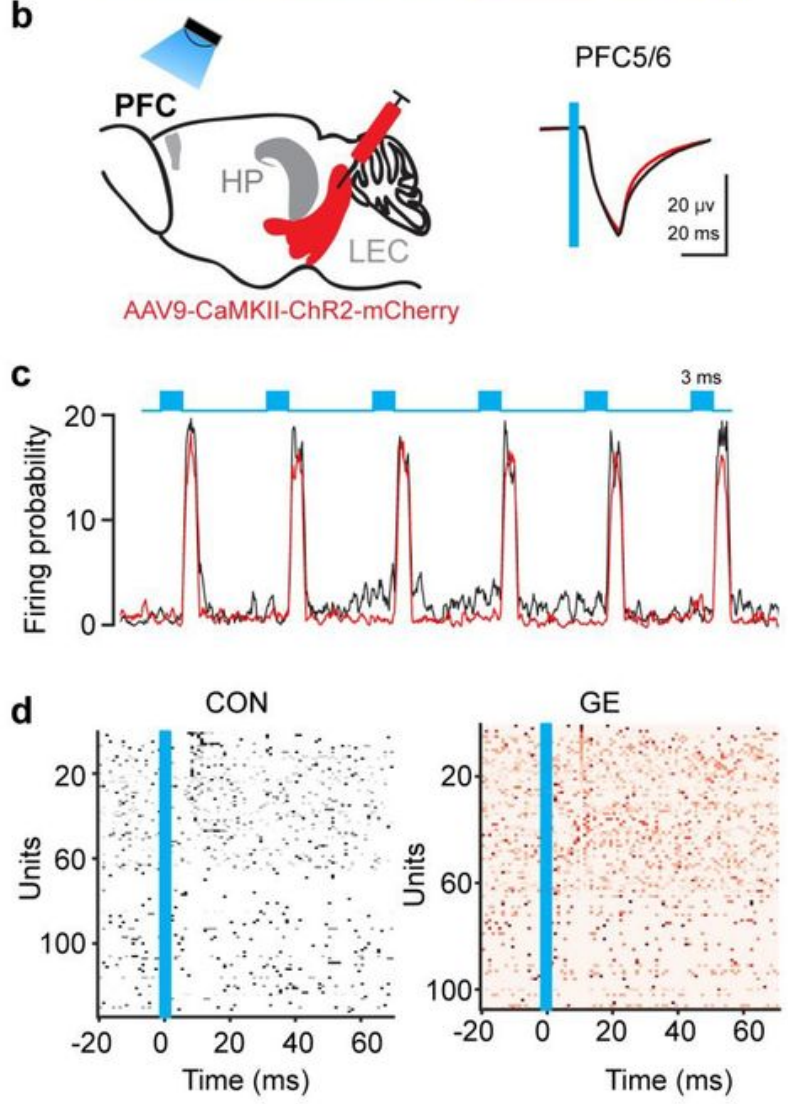

Figure 5 
Prelimbic neurons innervated by LEC and light-induced activation of LEC terminals in PFC. (a) Schematic of the anterograde trans-synaptic WGA injection in LEC. Photograph depicting the injection position in LEC of a P10 CON mouse. Bottom left, photographs depicting WGA-expressing neurons (green dots) in the PFC of a P10 CON mouse. Bottom right, WGA-labeled neurons in prelimbic layers displayed at a higher-magnification. PL, prelimbic subdivision of the PFC; IL, infralimbic subdivision of the PFC. (b) Schematic of the AAV9-CaMKII-ChR2-mCherry injection in the LEC and light stimulation of entorhinal axonal terminals in PFC. Right, averaged LFP traces recorded in the prelimbic layer $5 / 6$ in response tolight stimulation of LEC terminals in CON (black) and GE (red) mice. The blue line indicates the 3 ms-long pulse stimulation in PFC. (c) Spike probability histogram of prelimbic layer $5 / 6$ neurons in response to 120 sweeps of $8 \mathrm{~Hz}$ pulse stimulation (3 ms pulse length, $473 \mathrm{~nm}$, blue line) in CON (black) and GE mice (red). (d) Raster plot depicting the firing of single cells in response to the first pulse stimulation from each sweep in CON and GE groups. The blue line corresponds to the 3 ms-long pulse stimulation in PFC. 

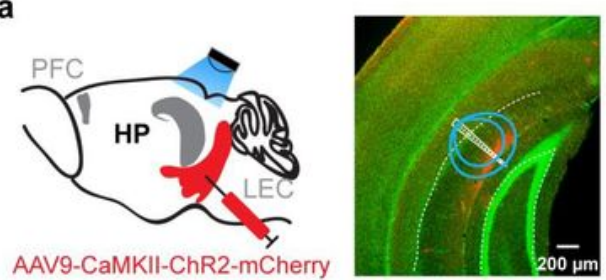

b
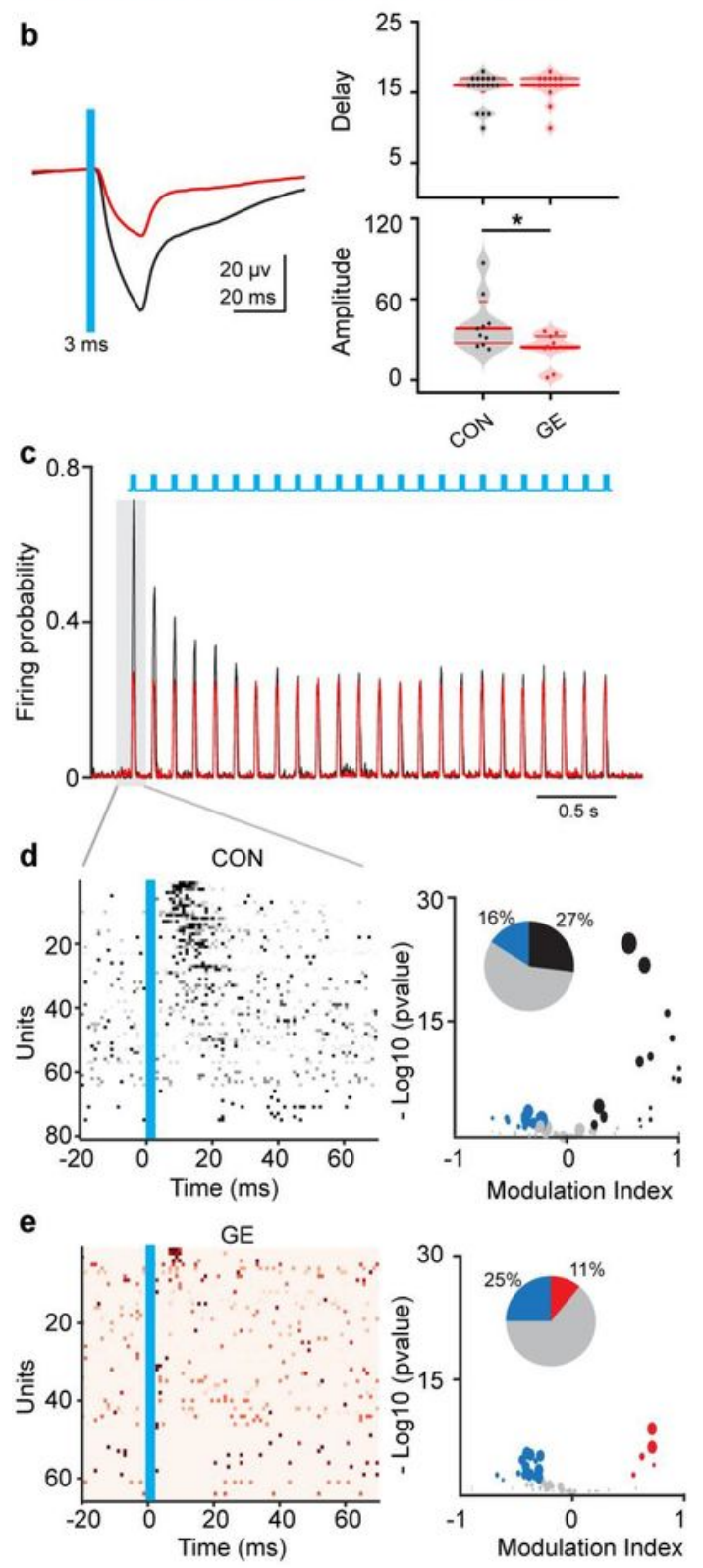

Figure 6

Light-induced activation of LEC terminals in HP. (a) Schematic of the AAV9-CaMKII-ChR2-mCherry injection in LEC and light stimulation of entorhinal axonal terminals in HP. Right, photographs depicting the LEC axonal terminals labeled by mCherry (red) in the HP of a P9 CON mouse. Blue lines correspond to the iso-contour lines for light power of 1 and $10 \mathrm{~mW} / \mathrm{mm} 2$, respectively. (b) Averaged LFP traces recorded in the HP in response to light stimulation of LEC terminals in CON (black) and GE (red) mice. The blue line 
indicates the $3 \mathrm{~ms}$ - long pulse stimulation in HP. Right up, violin plots displaying the delay of the biggest response of the averaged LFP in HP. Right bottom, violin plots displaying the amplitude of the biggest response of the averaged LFP in HP. (c) Spike probability histogram for hippocampal neurons in response to 120 sweeps of $8 \mathrm{~Hz}$ pulse stimulation (3 ms pulse length, $473 \mathrm{~nm}$, blue line) in CON (black) and GE (red) mice. (d) Left, raster plot depicting the firing of single hippocampal cells in response to the first pulse stimulation from each sweep in CON group. Right, the modulation index of spiking response of hippocampal single units to pulse stimulation. Modulation index $>0$ indicates increased firing activity, whereas values $<0$ correspond to decreased firing activity. Inset, pie plot depicting the percentage of hippocampal single units activated (black), inhibited (blue), non-responded (gray) to the stimulation. (e) Same as (d) for GE mice. Single data points are shown as dots and the red horizontal bars in violin plots 9 correspond to the median and the 25th and 75th percentiles. ${ }^{*} p<0.05$.

a

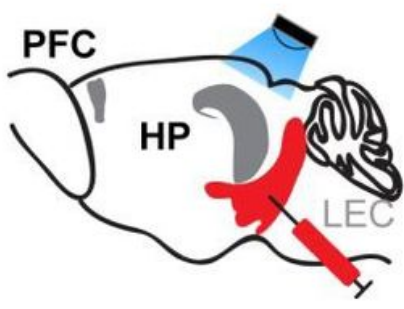

AAV9-CaMKII-ChR2-mCherry

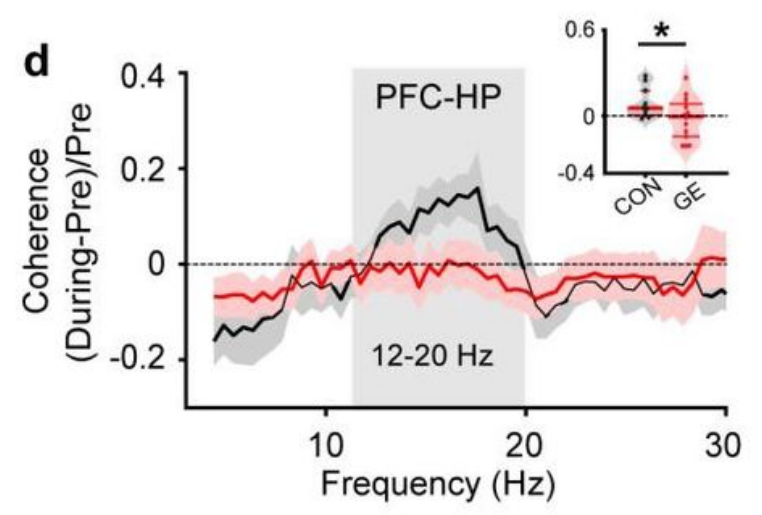

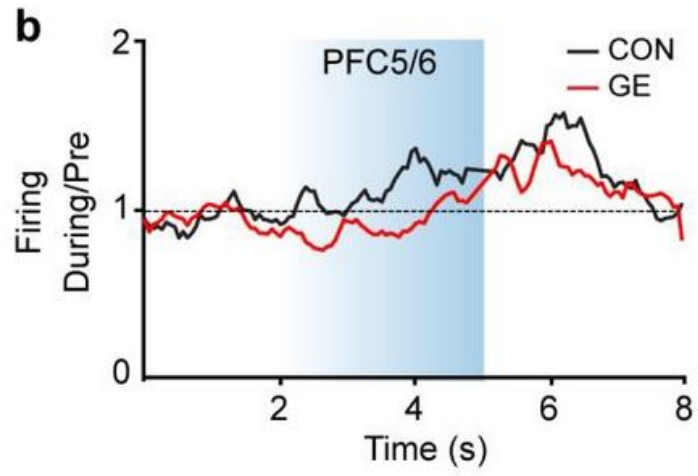
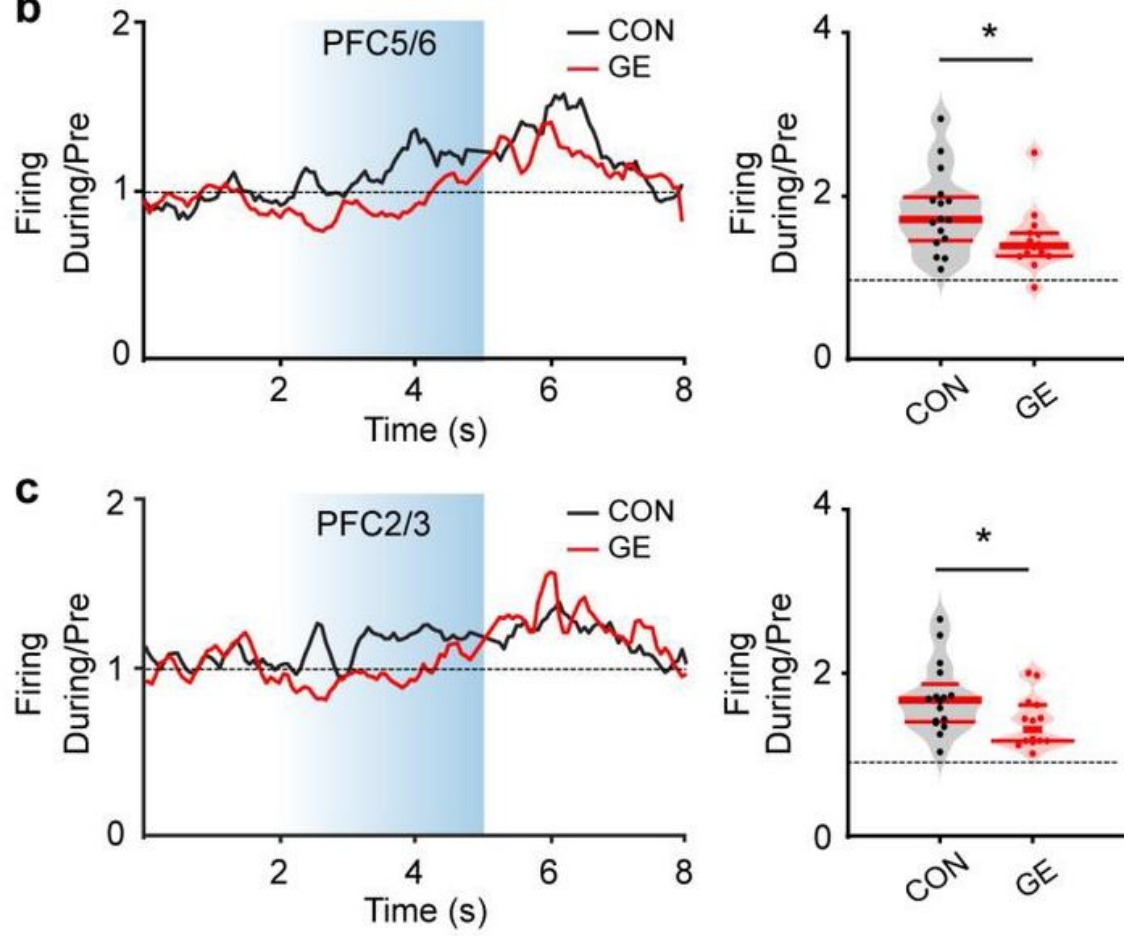

\section{Figure 7}

Firing activity in PFC during light-induced activation of LEC terminals in HP. (a) Schematic of AAV9CaMKII-ChR2-mCherry injection in LEC and light stimulation of entorhinal axonal terminals in HP. (b) Line plot of firing activity of prelimbic layer $5 / 6$ neurons during 3 s-long ramp stimulation (light blue shadow) of LEC terminals in HP normalized to the activity before stimulation for CON and GE mice. The horizontal dotted line corresponds to no changes of firing activity during the stimulation. Right, violin plots displaying the firing activity of prelimbic layer $5 / 6$ neurons during $3 \mathrm{~s}$-long ramp stimulation normalized to the activity before stimulation. (c) Same as (b) for prelimbic layer 2/3 neurons. (d) Line plots of HP-PFC coherence calculated during ramp stimulation of entorhinal terminals in HP and normalized to coherence values before stimulation. Inset, violin plot displaying the averaged $12-20 \mathrm{~Hz}$ coherence between HP and 
PFC during stimulation when normalized to coherence values before stimulation. Single data points are shown as dots and the red horizontal bars in violin plots correspond to the median and the 25th and 75th percentiles. ${ }^{\star} \mathrm{p}<0.05,{ }^{\star \star} \mathrm{p}<0.01$.

\section{Supplementary Files}

This is a list of supplementary files associated with this preprint. Click to download.

- Supplementaryinformation.pdf 